\title{
The Gaia-ESO Survey: the origin and evolution of s-process elements ${ }^{\star}$
}

L. Magrini ${ }^{1}$, L. Spina ${ }^{2}$, S. Randich ${ }^{1}$, E. Friel $^{3}$, G. Kordopatis ${ }^{4}$, C. Worley ${ }^{5}$, E. Pancino ${ }^{1,6}$, A. Bragaglia ${ }^{7}$, P. Donati ${ }^{7}$, G. Tautvaišiené ${ }^{8}$, V. Bagdonas ${ }^{8}$, E. Delgado-Mena ${ }^{9}$, V. Adibekyan ${ }^{9}$, S. G. Sousa ${ }^{9}$, F. M. Jiménez-Esteban ${ }^{10}$, N. Sanna ${ }^{1}$, V. Roccatagliata ${ }^{1}$, R. Bonito ${ }^{11}$, L. Sbordone ${ }^{12}$, S. Duffau ${ }^{13}$, G. Gilmore ${ }^{5}$, S. Feltzing ${ }^{14}$, R. D. Jeffries ${ }^{15}$, A. Vallenari ${ }^{16}$, E. J. Alfaro ${ }^{17}$, T. Bensby ${ }^{14}$, P. Francois $^{18}$, S. Koposov ${ }^{5}$, A. J. Korn ${ }^{19}$, A. Recio-Blanco ${ }^{20}$, R. Smiljanic ${ }^{21}$, A. Bayo ${ }^{22,23}$, G. Carraro ${ }^{24}$, A. R. Casey $^{2}$, M. T. Costado ${ }^{25}$, F. Damiani ${ }^{11}$, E. Franciosini ${ }^{1}$, A. Frasca ${ }^{26}$, A. Hourihane ${ }^{5}$, P. Jofré ${ }^{27}$, P. de Laverny ${ }^{20}$, J. Lewis ${ }^{5}$, T. Masseron ${ }^{28,29}$, L. Monaco ${ }^{13}$, L. Morbidelli ${ }^{1}$, L. Prisinzano ${ }^{11}$, G. Sacco ${ }^{1}$, and S. Zaggia ${ }^{16}$

(Affiliations can be found after the references)

Received 16 February 2018 / Accepted 5 June 2018

\section{ABSTRACT}

Context. Several works have found an increase of the abundances of the $s$-process neutron-capture elements in the youngest Galactic stellar populations. These trends provide important constraints on stellar and Galactic evolution and they need to be confirmed with large and statistically significant samples of stars spanning wide age and distance intervals.

Aims. We aim to trace the abundance patterns and the time evolution of five $s$-process elements - two belonging to the first peak, $\mathrm{Y}$ and $\mathrm{Zr}$, and three belonging to the second peak, $\mathrm{Ba}$, La, and $\mathrm{Ce}$ - using the Gaia-ESO IDR5 results for open clusters and disc stars.

Methods. From the UVES spectra of cluster member stars, we determined the average composition of clusters with ages $>0.1$ Gyr. We derived statistical ages and distances of field stars, and we separated them into thin and thick disc populations. We studied the time-evolution and dependence on metallicity of abundance ratios using open clusters and field stars whose parameters and abundances were derived in a homogeneous way. Results. Using our large and homogeneous sample of open clusters, thin and thick disc stars, spanning an age range larger than $10 \mathrm{Gyr}$, we confirm an increase towards young ages of $s$-process abundances in the solar neighbourhood. These trends are well defined for open clusters and stars located nearby the solar position and they may be explained by a late enrichment due to significant contribution to the production of these elements from long-living low-mass stars. At the same time, we find a strong dependence of the s-process abundance ratios on the Galactocentric distance and on the metallicity of the clusters and field stars.

Conclusions. Our results, derived from the largest and most homogeneous sample of $s$-process abundances in the literature, confirm the growth with decreasing stellar ages of the $s$-process abundances in both field and open cluster stars. At the same time, taking advantage of the abundances of open clusters located in a wide Galactocentric range, these results offer a new perspective on the dependence of the $s$-process evolution on the metallicity and star formation history, pointing to different behaviours at various Galactocentric distances.

Key words. Galaxy: abundances - open clusters and associations: general - Galaxy: disk

\section{Introduction}

Elemental abundances and abundance ratios in the different populations of our Galaxy provide fundamental constraints on the scenarios of galaxy formation and evolution. With a large number of different elements we can study the different processes and time scales involved in stellar and Galactic evolution.

The heavy elements (atomic number $Z>30$ ) are produced by successive capture of neutrons by lighter elements. Their evolution in the Galaxy has been studied since the pioneering work of Pagel \& Tautvaisiene (1997) and Travaglio et al. (1999). There are 54 stable or long-lived neutron-capture elements, compared to only 30 lighter elements (cf. Sneden et al. 2008). However, they are much less abundant than the lighter elements and correspond to an abundance by number of $10^{-8}$ in the Sun. Their production is an endothermic process. In addition, the Coulomb barriers increase with the proton number. Consequently, the nuclei heavier than iron can be created only with a series of

\footnotetext{
* Based on observations collected with the FLAMES instrument at VLT/UT2 telescope (Paranal Observatory, ESO, Chile), for the GaiaESO Large Public Spectroscopic Survey (188.B-3002, 193.B-0936).
}

neutron capture events: for those events, there are no Coulomb barriers. Neutron-capture elements can be split into two different groups: those formed mainly via the slow neutron-capture "s-process" and those produced by the rapid neutron capture " $r$-process". The terms slow (timescale for a single neutron capture of hundreds or thousands of years) or rapid (fractions of second) are so defined in comparison with the timescales of the $\beta$-decay of the nuclei onto which neutrons are accreted. For the $s$-process and $r$-process, low, that is, $10^{7}-10^{11} \mathrm{~cm}^{-2} \mathrm{~s}^{-1}$, and high, $\sim 10^{20} \mathrm{~cm}^{-2} \mathrm{~s}^{-1}$, fluxes of neutrons are needed, respectively.

The $s$-process-dominated elements, hereafter $s$-process elements, are distributed primarily in two peaks in the periodic table around the neutron magic numbers $N=50,82$. The first $s$ process peak is located around $N=50$ and leads to the formation of $\mathrm{Sr}$, Y, and $\mathrm{Zr}$ (light-s elements); the second peak at $N=82$ produces $\mathrm{Ba}, \mathrm{La}, \mathrm{Ce}, \mathrm{Pr}$, and $\mathrm{Nd}$. There is also a third peak that corresponds to the production of $\mathrm{Pb}$ at $N=126$. The $s$-process can be further divided into a "main" process, a "weak" process and a "strong" process: the first occurs during the asymptotic giant branch (AGB) phases (Gallino et al. 1998; Busso et al. 1999), whereas the second takes place in massive stars that produce 
elements with $N \leq 88$ (Raiteri et al. 1993; Pignatari et al. 2010) and the third is responsible for about $50 \%$ of solar ${ }^{208} \mathrm{~Pb}$ production by low-metallicity AGB stars (e.g. Gallino et al. 1998).

Recently, several works found an increase of the abundances of the $s$-process elements for the youngest stellar populations. Among the first studies to notice this increase, D'Orazi et al. (2009) reported the discovery of increasing barium abundance with decreasing age for a large sample of Galactic open clusters. They suggested a first tentative explanation assuming a higher Ba yield from the $s$-process in low-mass stars than the average suggested by parameterised models of neutron-capture nucleosynthesis. However, their chemical evolution model was not able to explain the very high $[\mathrm{Ba} / \mathrm{Fe}]$ measured in the youngest open clusters of their sample. Other studies followed, all finding strong trends of $\mathrm{Ba}$ abundance with age.

A number of studies (e.g. Maiorca et al. 2011; Jacobson \& Friel 2013; Mishenina et al. 2013, 2015) extended the analysis to other $s$-process elements, both lighter (Y, Zr) and heavier (Ba, La, Ce) neutron-rich nuclei. Conclusions varied with the studies, painting a somewhat confused picture of age dependencies in elements other than Ba. Maiorca et al. (2011) found the elements $\mathrm{Y}, \mathrm{Ce}, \mathrm{Zr}$, and $\mathrm{La}$ to show increasing abundance ratios with decreasing age, as with $\mathrm{Ba}$, but to a lesser degree. Jacobson \& Friel (2013) found strong age trends in Ba but no age trends in $\mathrm{Zr}$ and $\mathrm{La}$, and noted that the conclusions drawn by different studies depended on the sample size and the range of ages sampled, and were affected by sometimes large systematic differences between studies (e.g. Yong et al. 2012). Mishenina et al. (2013, 2015) found clusters and field stars shared similar behaviour in $\mathrm{Y}$ and La, with slight, if any, trends with age. A number of studies found overall enhancements in abundance ratios in the clusters relative to solar, up to $0.2 \mathrm{dex}$, particularly for $[\mathrm{Zr} / \mathrm{Fe}]$.

Clusters in these samples not only spanned large age ranges, from several hundred million years to $\sim 8 \mathrm{Gyr}$, but they also covered large distance ranges, in particular sampling the outer disc. Although open clusters have been shown not to follow any relationship between age and metallicity, they do exhibit gradients in abundance, and their location (at birth) is more of a factor in determining their abundance than their age is. The interplay of the effects of both age and location introduce complexity into the interpretation of abundance trends, while at the same time offering the potential to illuminate them. While these cluster studies benefited from the accurate age determinations of open clusters, particularly their ability to trace the recent epoch, they nevertheless revealed that differing sample sizes, age, and distance distributions, and the non-uniformity of analysis between studies complicated the conclusions that could be drawn, particularly regarding weak trends.

Other groups investigated this phenomenon using field stars, especially solar twins. We recall that the ages of field stars can be determined with lower accuracy than those for open clusters at least in the pre-Gaia era. The sample of solar-type stars of da Silva et al. (2012) showed a pattern of [s/Fe] with age similar to that of open clusters (D'Orazi et al. 2009; Maiorca et al 2012), with increasing abundance ratios with decreasing age, although the form of the dependence varied with element: for $\mathrm{Ce}$ and $\mathrm{Zr}$, the increase pertained only to stars younger than the Sun, while for $\mathrm{Y}$ and $\mathrm{Sr}$, the abundances increased linearly with decreasing age. Recently, Reddy \& Lambert (2017) measured La, $\mathrm{Ce}, \mathrm{Nd}$, and Sm: their Fig. 7 suggests that the $[\mathrm{X} / \mathrm{Fe}]$ values measured for these heavy $s$-process elements display a smoothly increasing weak trend with decreasing stellar age, with changes of $\sim 0.1$ dex from $10 \mathrm{Gyr}$ ago to the present. For Ba, they found, like many others, a much stronger age dependence. They explain the larger enhancement for $\mathrm{Ba}$ in younger stars as due to an overestimation of $\mathrm{Ba}$ by standard methods of Local Thermodynamical Equilibrium (LTE) analysis, finding the Ba enhancements to be strongly correlated with the level of chromospheric activity. If this effect is considered and the very young stars are excluded (the chromospheric activity has a striking decline from 1-10 Myr to the age of the Sun), the enhancement of $\mathrm{Ba}$ is in line with that of the other $s$-process elements. In the same framework, Nissen et al. (2017) analysed a sample of ten stars from the Kepler LEGACY (Silva Aguirre et al. 2017) and found that over the lifetime of the Galactic disc the abundance of $\mathrm{Y}$ increases. The trend of $[\mathrm{Y} / \mathrm{Fe}]$ with age, and an even stronger trend of [Y/Mg], were previously found by, for example da Silva et al. (2012), Nissen (2016), Spina et al. (2016), Feltzing et al. (2017) and it can be explained by production of yttrium via the slow neutron-capture process in low-mass AGB stars (Maiorca et al. 2012; Karakas \& Lugaro 2016). Finally, Spina et al. (2018), in their differential analysis of a sample of solar twin stars, found an increase with decreasing stellar ages of the abundance ratios $[\mathrm{X} / \mathrm{Fe}]$ of all the $s$-process elements of the first and second peaks. They found that the rise of $\mathrm{Ba}$ with time is consistent with that of the other n-capture elements, considering its higher contribution from the $s$-process. In all cases, trends of $[\mathrm{X} / \mathrm{Fe}]$ are weak, with changes of $\sim 0.1$ to $\sim 0.2$ dex over the lifetime of the disc.

Therefore, a general consensus of an increasing, though weak, trend in the $s$-process elements production exists. There are still issues regarding the maximum abundance for $\mathrm{Ba}$, which is still debated (see Reddy \& Lambert 2017) and the trends for the open clusters, which are affected by positional dependence, radial gradients, and differences in analysis and samples (see, e.g. Jacobson et al. 2011; Yong et al. 2012). Taking inspiration from the conclusions of Jacobson \& Friel (2013), we need a homogeneous analysis of large samples of open clusters together with a sample of field stars that will facilitate the measurement of many $n$-capture species in clusters and in the field spanning a wide range in age.

Among the several on-going spectroscopic surveys, the GaiaESO Survey (GES, Gilmore et al. 2012; Randich et al. 2013), an ESO large public survey, is providing high-resolution spectra of different stellar populations of our Galaxy using FLAMES at VLT (Pasquini et al. 2002) and employing both Giraffe and UVES fibres in Medusa mode. This survey aims at homogeneously determining stellar parameters and abundances for a large sample of stars in the field, in Galactic open clusters, and in calibration samples, including also globular clusters. In particular, the high-resolution spectra obtained with UVES allow the determination of abundances of a large variety of elements including many neutron-capture elements: two light $s$-process (ls) elements (Y, $\mathrm{Zr}$ ), three $s$-process (hs) elements (Ba, La, Ce), one $r$-process element $(\mathrm{Eu})$, and three elements with significant contributions from both processes (mixed elements - Mo, Pr, and Nd). In the present work, we investigate the evolution of the group of $s$-process elements. The paper is structured as follows. In Sect. 2, we present the data reduction and analysis and in Sect. 3 the solar abundance scale. In Sect. 4 we describe the field and cluster samples, in Sect. 5 we show the results on the abundance of $s$-process elements, and in Sect. 6 we discuss their time evolution. Finally, in Sect. 7 we give our summary and conclusions.

\section{The data reduction and analysis}

We use data from the fifth internal data release (IDR5) of the Gaia-ESO Survey. For this work we use only the products based 
on the UVES spectra, which have a higher spectral resolution $(R=47000)$ and a wider spectral range. They have been reduced and analysed by the Gaia-ESO consortium. UVES data reduction is carried out using the FLAMES-UVES ESO public pipeline (Modigliani et al. 2004). The UVES data reduction process and the determination of the radial velocities (RVs) are described in Sacco et al. (2014). Various Working Groups (WGs) contribute to the spectral analysis of different kinds of stars and/or setups: the data discussed in the present paper have been analysed by WG11 which is in charge of the analysis of the UVES spectra of F-G-K spectral-type stars both in the field of the Milky Way (MW) and in intermediate-age and old clusters and obtained with the UVES red arm standard setting with central wavelengths of 520 and $580 \mathrm{~nm}$. These spectra were analysed with the Gaia-ESO multiple pipelines strategy, as described in Smiljanic et al. (2014). The results of each pipeline are combined with an updated methodology (Casey et al., in prep.) to define a final set of recommended values of the atmospheric parameters. The results of WG11 are homogenised using several calibrators, for example benchmark stars and open/globular clusters selected as described in Pancino et al. (2017) and adopted for the homogenisation by WG15 (Hourihane et al., in prep.). The recommended parameters and abundances used in the present work are reported in the final IDR5 catalogue, which contains the observations obtained before December 2015.

To measure the usually faint absorption lines of neutron capture elements, a high signal-to-noise ratio $(\mathrm{S} / \mathrm{N})$ spectrum is required. For this reason, for the field stars, we made a selection of the UVES results based on the S/N and on the quality of the parameter determination. The thresholds of our cuts are defined comparing our results with the very high-S/N samples of Bensby et al. (2014) for Ba and of Battistini \& Bensby (2016) for the other elements, designed to study the neutroncapture element abundances. To obtain similar dispersions to those of Bensby et al. (2014) and Battistini \& Bensby (2016) for the neutron-capture element distributions (at a given metallicity) we need a minimum S/N of 70 for Milky Way field stars. In addition, we discard stars whose errors on stellar parameters are: $E T_{\text {eff }}>150 \mathrm{~K}, E_{\log g}>0.25 \mathrm{dex}, E_{[\mathrm{Fe} / \mathrm{H}]}>0.20 \mathrm{dex}$ and $E_{\xi}>0.20 \mathrm{~km} \mathrm{~s}^{-1}$. The final sample of field stars fulfilling these requirements includes about 600 objects. The sample of open clusters includes 22 clusters, with a total of 165 member stars.

For the cluster stars, we did not apply any selection, because their spectra are usually characterised by higher $\mathrm{S} / \mathrm{N}$ (and therefore almost all stars in cluster stars will be included). In addition, we do not consider individual measurements for cluster stars, but we rely on the cluster median abundances, which are derived from several member stars (the membership analysis is discussed in Sect. 4.2). We only discard cluster abundances with high standard deviations $>0.3$ dex.

\section{Solar abundance scale}

To obtain abundances on the solar scale, we need to define our abundance reference. In Table 1 we show three different sets of abundances: the solar abundances from IDR5 computed from archive solar spectra; those from Grevesse et al. (2007); and the abundances of giant stars in M67. The abundances are in the usual $12+\log (\mathrm{X} / \mathrm{H})$ form. The cluster M67 is known to have the same composition as the Sun (e.g. Randich et al. 2006; Pasquini et al. 2008; Önehag et al. 2014; Liu et al. 2016) and therefore it is useful to confirm it with the IDR5 data. Since small effects of stellar diffusion might be present in the abundances of the dwarf stars of M67 (see, e.g. Önehag et al. 2014;
Table 1. IDR 5 solar and M67 abundances.

\begin{tabular}{llll}
\hline \hline Element & Sun (IDR5) & $\begin{array}{l}\text { Sun } \\
\text { (Grevesse et al. } \\
\text { 2007) }\end{array}$ & M67 giants (IDR5) \\
\hline Y II & $2.19 \pm 0.12$ & $2.21 \pm 0.02$ & $2.14 \pm 0.01(0.09)$ \\
Zr II & $2.53 \pm 0.13$ & $2.58 \pm 0.02$ & $2.54 \pm 0.03(0.05)$ \\
Ba II & $2.17 \pm 0.06$ & $2.17 \pm 0.07$ & $2.07 \pm 0.03(0.07)$ \\
La II & - & $1.13 \pm 0.05$ & $1.00 \pm 0.02(0.12)$ \\
Ce II & $1.70 \pm 0.11$ & $1.70 \pm 0.10$ & $1.71 \pm 0.01(0.01)$ \\
Eu II & - & $0.52 \pm 0.06$ & $0.42 \pm 0.01(0.04)$ \\
\hline
\end{tabular}

Bertelli Motta et al. 2018), we only considered the abundances of the giant stars. The GES solar abundances for the $s$-process elements are in very good agreement with the reference solar abundances from Grevesse et al. (2007). The average abundances for the three member giant stars in M67 ( $T_{\text {eff }} \sim 4800-4900$ and $\log g \sim 3-3.4)$ from the IDR5 recommended values are given together with their standard deviations of the mean and the typical errors on each measurement (in parenthesis; see fourth column of Table 1). In the following, we normalise our abundances to the solar abundances (second column), with the exception of La for which there is no solar abundance available and therefore we make use of the M67 abundances.

\section{The stellar samples}

\subsection{The Milky Way field samples}

The sample discussed here includes field stars observed with UVES 580 that belong to the stars in the Milky Way sample, and in particular to the solar neighbourhood sample (GES_TYPE = "GE_MW") and the inner disc sample (GES_TYPE = "GE_MW_BL"). The selection functions of these stars are described in Stonkute et al. (2016). For these stars, we computed spectroscopic distances as in Magrini et al. (2017), by projecting the stellar atmospheric parameters and (J-K) 2MASS colours on a set of isochrones (Bressan et al. 2012) spanning the age range between 0.1 and $13.9 \mathrm{Gyr}$ (with a uniform step of $0.1 \mathrm{Gyr}$ ) and a metallicity range between -2.3 and +0.2 dex (with a uniform step of $0.1 \mathrm{dex}$ ). The projection takes into account the uncertainties on the atmospheric parameters, the likelihood of a star to belong to a given evolutionary phase, and the line-of-sight extinction (iteratively corrected by the distance) of Schlegel et al. (1998). The projection therefore not only returns the absolute magnitude in various bands (and therefore, eventually, the line-of-sight distance), but also a crude estimation of the stellar ages. The details of the method are described in Kordopatis et al. (2011), with the updates of Recio-Blanco et al. (2014) and Kordopatis et al. (2015). Typical errors range from $20 \%$ up to more than $100 \%$, with the bulk of the stars having errors of the order of $30-50 \%$.

We have divided the field stars into thin- and thick-disc stars, using their $[\alpha / \mathrm{Fe}]$ abundance ratio ${ }^{1}$ to discriminate the two populations, following the approach of Adibekyan et al. (2011). We have used the multi-slope curve of Adibekyan et al. (2011) to define thin- and thick-disc stars on the basis of their $[\alpha / \mathrm{Fe}]$ and $[\mathrm{Fe} / \mathrm{H}]$. In the following analysis, we have included stars with $\mathrm{T}_{\text {eff }}<6120 \mathrm{~K}$ (to avoid NLTE effects in some neutron-capture element abundances as discussed in Bensby et al. 2014) and with

${ }^{1}[\alpha / \mathrm{Fe}]$ is computed by averaging $[\mathrm{Mg} / \mathrm{Fe}],[\mathrm{Ca} / \mathrm{Fe}],[\mathrm{Si} / \mathrm{Fe}]$ and excluding $[\mathrm{O} / \mathrm{Fe}]$, which is affected by large errors in dwarf stars. 
$\mathrm{S} / \mathrm{N}>70$, and applied the error cuts described in the previous section (see Sect. 2) to exclude poor-quality results. The adopted separation between thin- and thick-disc stars is also presented in the first panels of Figs. 3 and 4. In the following discussion, we include only thin- and thick-disc stars located in the solar neighbourhood $\left(6.5 \mathrm{kpc}<R_{\mathrm{GC}}<9.5 \mathrm{kpc}\right)$ to avoid confusion due to the radial dependence of the abundance ratios.

\subsection{The cluster sample}

We consider the sample of open clusters with ages $>0.1 \mathrm{Gyr}$ whose parameters and abundances have been delivered in IDR5. We do not include younger clusters because their stars are usually more difficult to analyse and they can be affected by chromospheric effects.

For each cluster, we have extracted member stars using the information on their radial velocities. We have considered as member stars those within 1.5- $\sigma$ from the cluster systemic velocity (computed using the UVES spectra) and we have excluded outliers in metallicity $\left|[\mathrm{Fe} / \mathrm{H}]_{\mathrm{star}}-<[\mathrm{Fe} / \mathrm{H}]>\right|>0.1$ dex. Based on stars assigned as members, we have computed the median elemental abundances, expressed in the form $[\mathrm{X} / \mathrm{H}]=\log (\mathrm{X} / \mathrm{H})-\log (\mathrm{X} / \mathrm{H})_{\odot}$, which are presented in Table 2 . The uncertainties reported on each abundance are the standard deviation of cluster member abundances. In the following analysis, we only consider abundance ratios with a dispersion lower than 0.3 dex.

In Table 2, we summarise the basic properties of the sample open clusters: coordinates, ages, Galactocentric distances, heights above the plane, mean radial velocities of cluster members, median metallicity and the number of cluster-member stars used to compute the metallicity. There is a general agreement with the previous official release (see, e.g. Magrini et al. 2017), but for some clusters the median metallicity might have changed slightly. For clusters in common with Magrini et al. (2017), we adopt the same ages and distances, and for the clusters that are new in IDR5, we report ages and distances from the recent literature.

In Tables 3 and 4 , we present the median abundances $[\mathrm{X} / \mathrm{H}]$ and abundance ratios, $[\mathrm{X} / \mathrm{Fe}]=\left[\log (\mathrm{X} / \mathrm{H})-\log (\mathrm{X} / \mathrm{H})_{\odot}\right)-$ $\left.\left(\log (\mathrm{FeI} / \mathrm{H})-\log (\mathrm{FeI} / \mathrm{H})_{\odot}\right)\right]$. The latter may slightly differ from the simple subtraction of the median $[\mathrm{X} / \mathrm{H}]$ and $[\mathrm{Fe} / \mathrm{H}]$, because the mean metallicity $[\mathrm{Fe} / \mathrm{H}]$ of each cluster is computed from the global metallicity (FEH column of IDR5), while we have computed $[\mathrm{X} / \mathrm{Fe}]$ using the iron abundance derived from neutral iron lines (FE1 column). In principle, for elements with singly ionised atoms it would be more appropriate to use FeII abundances to obtain their abundance ratios, $[\mathrm{X} / \mathrm{Fe}]$. We have checked the consistency between $\log (\mathrm{Fe} / \mathrm{H})$ and $\log (\mathrm{FeII} / \mathrm{H})$ in our sample, finding an excellent agreement, with a small systematic offset $\log (\mathrm{FeII} / \mathrm{H})-\log (\mathrm{Fe} / \mathrm{H}) \sim 0.05$ dex which might systematically lower our results by this small amount. However, since the FeII abundances are affected, on average, by larger errors due to the smaller number of FeII lines than of FeI lines, we adopted the FeI abundances to compute the $[\mathrm{X} / \mathrm{Fe}]$ abundance ratios. The choice of $\mathrm{FeI}$ to compute $[\mathrm{X} / \mathrm{Fe}]$ does not affect the trends or relative comparisons within the Gaia-ESO samples.

\section{The abundances of the s-process elements}

Following the literature and, in particular, Overbeek et al. (2016), we divide the neutron capture elements into $s$-elements, those dominated by $s$-process if at least $70 \%$ of their abun- dance in the Sun is produced by the $s$-process, and, vice versa, $r$-elements, those dominated by the $r$-process for more than $70 \%$ in the Sun. Elements whose nucleosynthesis is not dominated by either the $s$ - or $r$-process are defined as mixed elements. We follow the review of Sneden et al. (2008) for what concerns the isotopic composition, the mixture of $s$ - and $r$-processes in the Sun and their relative percentages, but we compare also with the recent redetermination of the solar composition by Bisterzo et al. (2014). The isotopic composition and (within parenthesis) the total percentage in the Sun from Sneden et al. (2008) are reported, for each element, in Table 5. In the last column, we also report the total percentage of each element in the Sun from Bisterzo et al. (2014).

\subsection{The effect of the evolutionary stage: comparing abundances in dwarf and giant stars among cluster-member stars}

In a purely differential analysis, with respect to the Sun, of stars in open clusters, D'Orazi et al. (2009) showed that [Ba/Fe] ratios are systematically higher in giant stars than in dwarf stars in a sample of clusters where both kinds of star have been analysed. This effect is related to the use of a dwarf star, the Sun, as a reference in the analysis of giant stars.

We have analysed the abundance ratios $[\mathrm{X} / \mathrm{Fe}]$ as a function of the surface gravity in member stars of clusters in which both dwarf and giant stars were observed. The results of [Y/Fe], $[\mathrm{Zr} / \mathrm{Fe}],[\mathrm{Ba} / \mathrm{Fe}]$ and $[\mathrm{Ce} / \mathrm{Fe}]$ for NGC 6633, NGC 2420, and M67 are shown in Fig. 1. The behaviour of lanthanum is not shown since IDR 5 contains La abundances for almost exclusively giant stars. The aim of Fig. 1 is to highlight possible differences in the abundances of the $s$-process elements due to the different stellar parameters. In these plots, we have normalized the abundance ratios of the member stars of each cluster to its median abundance and have excluded stars with abundance uncertainties larger than 0.2 dex. From this figure, we do not observe, within the uncertainties, any systematic trends of the abundance ratios $[\mathrm{Y} / \mathrm{Fe}],[\mathrm{Zr} / \mathrm{Fe}]$, and $[\mathrm{Ce} / \mathrm{Fe}]$ with surface gravity for giant and dwarf member stars of the same cluster. However, for $[\mathrm{Ba} / \mathrm{Fe}]$, we notice that the stars with very low gravity in NGC 2420 have Ba abundance 0.2 dex higher than the other member stars. In the range of $\log \mathrm{g}$ from 2.5 to 4 there is no trend of $[\mathrm{Ba} / \mathrm{Fe}]$ versus $\log g$ and therefore no differences between dwarf and giant stars. To probe how much the overestimation of $\mathrm{Ba}$ abundance in low-gravity stars might be important, we have selected a sample of Milky Way field stars with $-0.1<[\mathrm{Fe} / \mathrm{H}]<0.1$. In Fig. 2, we plot their $[\mathrm{Ba} / \mathrm{Fe}]$ versus $\log \mathrm{g}$, which indicates that there are no systematic differences between giant and dwarf stars. In addition, in our sample, the number of stars with very low gravity (stars with $\log g<2.5$ are $\sim 3 \%$ of the sample of Milky Way field stars) is very small and their eventual over-abundances of Ba should not influence our results.

As a side result, it is worth noting the presence of a peculiar star in NGC 2420: one of the member stars of NGC 2420 with $\mathrm{CNAME}=07382696+2133313$, has systematically higher abundances of $\mathrm{Y}, \mathrm{Zr}$, and $\mathrm{Ba}$. Its radial velocity is perfectly consistent with those of the other cluster members. However, in the HR diagram, it is located slightly outside the main locus of the Red Clump stars and also the abundances of some other elements are somewhat discrepant with respect to the average abundance of the cluster (e.g. $[\mathrm{O} / \mathrm{H}],[\mathrm{C} / \mathrm{H}],[\mathrm{Si} / \mathrm{H}],[\mathrm{Sc} / \mathrm{H}]$, and $[\mathrm{Ti} / \mathrm{H}]$ are about $-0.3,-0.1,-0.1,-0.15$, and -0.15 dex, respectively, lower than the average cluster values). Since its $[\mathrm{Fe} / \mathrm{H}]$ and radial 
Table 2. Open cluster parameters.

\begin{tabular}{|c|c|c|c|c|c|c|c|c|c|}
\hline Id & ${ }^{\mathrm{RA}}$ & $\begin{array}{r}\text { Dec } \\
000.0)\end{array}$ & $\begin{array}{l}\text { Age } \\
(\mathrm{Gyr})\end{array}$ & $\begin{array}{l}R_{\mathrm{GC}} \\
(\mathrm{kpc})\end{array}$ & $\begin{array}{c}Z \\
(\mathrm{pc})\end{array}$ & $\begin{array}{c}r v \\
\left(\mathrm{~km} \mathrm{~s}^{-1}\right) \\
\end{array}$ & $\begin{array}{c}{[\mathrm{Fe} / \mathrm{H}]} \\
(\mathrm{dex})\end{array}$ & n. m. & Ref. Age and Distance \\
\hline NGC 6067 & $16: 13: 11$ & $-54: 13: 06$ & $0.10 \pm 0.05$ & $6.81 \pm 0.12$ & $-55 \pm 17$ & $-39.0 \pm 0.8$ & $+0.20 \pm 0.08$ & 12 & Alonso-Santiago et al. (2017) \\
\hline NGC 2516 & 07:58:04 & $-60: 45: 12$ & $0.11 \pm 0.01$ & $7.98 \pm 0.01$ & $-97 \pm 4$ & $+23.6 \pm 1.0$ & $-0.06 \pm 0.05$ & 13 & Randich et al. (2018) \\
\hline NGC 6259 & $17: 00: 45$ & $-44: 39: 18$ & $0.21 \pm 0.03$ & $7.03 \pm 0.01$ & $-27 \pm 13$ & $+33.3 \pm 0.9$ & $+0.21 \pm 0.04$ & 11 & Mermilliod et al. (2001), Dias et al. (2002) \\
\hline NGC 3532 & $11: 05: 39$ & $-58: 45: 12$ & $0.30 \pm 0.10$ & $7.85 \pm 0.01$ & $+11 \pm 4$ & $+4.9 \pm 0.9$ & $-0.06 \pm 0.14$ & 3 & Clem et al. (2011) \\
\hline NGC 6705 & 18:51:05 & $-06: 16: 12$ & $0.30 \pm 0.05$ & $6.33 \pm 0.16$ & $-95 \pm 10$ & $+34.8 \pm 0.7$ & $+0.16 \pm 0.04$ & 16 & Cantat-Gaudin et al. (2014) \\
\hline NGC 6633 & $18: 27: 15$ & $+06: 30: 30$ & $0.52 \pm 0.10$ & $7.71 \pm 0.01$ & $+52 \pm 2$ & $-28.9 \pm 0.9$ & $-0.01 \pm 0.11$ & 10 & Randich et al. (2018) \\
\hline NGC 4815 & $12: 57: 59$ & $-64: 57: 36$ & $0.57 \pm 0.07$ & $6.94 \pm 0.04$ & $-95 \pm 6$ & $-29.7 \pm 0.5$ & $+0.11 \pm 0.01$ & 3 & Friel et al. (2014) \\
\hline $\operatorname{Tr} 23$ & 16:00:50 & $-53: 31: 23$ & $0.80 \pm 0.10$ & $6.25 \pm 0.15$ & $-18 \pm 2$ & $-61.3 \pm 0.9$ & $+0.21 \pm 0.04$ & 11 & Jacobson et al. (2016) \\
\hline Mel71 & $07: 37: 30$ & $-12: 04: 00$ & $0.83 \pm 0.18$ & $10.50 \pm 0.10$ & $210 \pm 20$ & $+50.8 \pm 1.3$ & $-0.09 \pm 0.03$ & 5 & Salaris et al. (2004) \\
\hline Be81 & 19:01:36 & $-00: 31: 00$ & $0.86 \pm 0.10$ & $5.49 \pm 0.10$ & $-126 \pm 7$ & $+48.2 \pm 0.5$ & $+0.22 \pm 0.07$ & 13 & Magrini et al. (2015) \\
\hline NGC 6802 & 19:30:35 & $+20: 15: 42$ & $1.00 \pm 0.10$ & $6.96 \pm 0.07$ & $+36 \pm 3$ & $+11.9 \pm 0.9$ & $+0.10 \pm 0.02$ & 8 & Jacobson et al. (2016) \\
\hline Rup134 & $17: 52: 43$ & $-29: 33: 00$ & $1.00 \pm 0.20$ & $4.60 \pm 0.10$ & $-100 \pm 10$ & $-40.9 \pm 0.6$ & $+0.26 \pm 0.06$ & 17 & Carraro et al. (2006) \\
\hline NGC 6005 & $15: 55: 48$ & $-57: 26: 12$ & $1.20 \pm 0.30$ & $5.97 \pm 0.34$ & $-140 \pm 30$ & $-23.5 \pm 1.0$ & $+0.19 \pm 0.02$ & 11 & Piatti et al. (1998) \\
\hline Pis 18 & 13:36:55 & $-62: 05: 36$ & $1.20 \pm 0.40$ & $6.85 \pm 0.17$ & $+12 \pm 2$ & $-27.5 \pm 0.6$ & $+0.22 \pm 0.04$ & 6 & Piatti et al. (1998) \\
\hline $\operatorname{Tr} 20$ & $12: 39: 32$ & $-60: 37: 36$ & $1.50 \pm 0.15$ & $6.86 \pm 0.01$ & $+136 \pm 4$ & $-40.1 \pm 1.0$ & $+0.15 \pm 0.07$ & 37 & Donati et al. (2014) \\
\hline Be44 & $19: 17: 12$ & $+19: 33: 00$ & $1.60 \pm 0.30$ & $6.91 \pm 0.12$ & $+130 \pm 20$ & $-8.8 \pm 0.5$ & $+0.27 \pm 0.06$ & 7 & Jacobson et al. (2016) \\
\hline NGC 2420 & 11:05:39 & $-58: 45: 12$ & $2.20 \pm 0.30$ & $10.76 \pm 0.20$ & $+765 \pm 50$ & $+4.9 \pm 0.9$ & $-0.13 \pm 0.04$ & 22 & Salaris et al. (2004), Sharma et al. (2006) \\
\hline $\mathrm{Be} 31$ & $06: 57: 36$ & $+08: 16: 00$ & $2.50 \pm 0.30$ & $15.16 \pm 0.40$ & $+340 \pm 30$ & $+57.5 \pm 0.9$ & $-0.27 \pm 0.06$ & 9 & Cignoni et al. (2011) \\
\hline $\mathrm{Be} 25$ & $06: 41: 16$ & $-16: 29: 12$ & $4.00 \pm 0.50$ & $17.60 \pm 1.00$ & $-1900 \pm 200$ & $+136.0 \pm 0.8$ & $-0.25 \pm 0.05$ & 6 & Carraro et al. (2005) \\
\hline NGC 2243 & $06: 29: 34$ & $-31: 17: 00$ & $4.00 \pm 1.20$ & $10.40 \pm 0.20$ & $-1200 \pm 100$ & $+60.2 \pm 0.5$ & $-0.38 \pm 0.04$ & 16 & Bragaglia \& Tosi (2006) \\
\hline M67 & $08: 51: 18$ & $+11: 48: 00$ & $4.30 \pm 0.50$ & $9.05 \pm 0.20$ & $+405 \pm 40$ & $+34.7 \pm 0.9$ & $-0.01 \pm 0.04$ & 19 & Salaris et al. (2004) \\
\hline Be36 & 07:16:06 & $-13: 06: 00$ & $7.00 \pm 0.50$ & $11.3 \pm 0.20$ & $-40 \pm 10$ & $+62.3 \pm 1.6$ & $-0.16 \pm 0.10$ & 7 & Donati et al. (2012) \\
\hline
\end{tabular}

Table 3. Abundance in open clusters, expressed in the form $[\mathrm{X} / \mathrm{H}]$.

\begin{tabular}{|c|c|c|c|c|c|c|}
\hline Id & [YII/H] & {$[\mathrm{ZrII} / \mathrm{H}]$} & {$[\mathrm{CeII} / \mathrm{H}]$} & {$[\mathrm{BaII} / \mathrm{H}]$} & [LaII/H] & {$[\mathrm{EuII} / \mathrm{H}]$} \\
\hline NGC 6067 & $0.19 \pm 0.05$ & $0.36 \pm 0.07$ & $0.13 \pm 0.07$ & $0.46 \pm 0.09$ & $0.20 \pm 0.06$ & $0.19 \pm 0.06$ \\
\hline NGC 2516 & $0.04 \pm 0.05$ & $0.52 \pm 0.06$ & $0.28 \pm 0.10$ & $0.17 \pm 0.08$ & - & - \\
\hline NGC 6259 & $0.14 \pm 0.03$ & $0.28 \pm 0.08$ & $0.09 \pm 0.12$ & $0.08 \pm 0.05$ & $0.13 \pm 0.11$ & $0.20 \pm 0.04$ \\
\hline NGC 3532 & $-0.09 \pm 0.24$ & - & - & $0.02 \pm 0.24$ & - & - \\
\hline NGC 6705 & $0.05 \pm 0.03$ & $0.22 \pm 0.06$ & $0.05 \pm 0.09$ & $0.20 \pm 0.11$ & $0.06 \pm 0.06$ & $0.15 \pm 0.05$ \\
\hline NGC 6633 & $0.01 \pm 0.04$ & $0.15 \pm 0.01$ & $0.09 \pm 0.02$ & $0.12 \pm 0.11$ & $0.06 \pm 0.03$ & $-0.03 \pm 0.13$ \\
\hline NGC 4815 & $0.08 \pm 0.07$ & $0.23 \pm 0.10$ & $0.06 \pm 0.08$ & $0.24 \pm 0.10$ & $0.09 \pm 0.07$ & $0.05 \pm 0.08$ \\
\hline $\operatorname{Tr} 23$ & $0.04 \pm 0.04$ & $0.22 \pm 0.04$ & $0.00 \pm 0.10$ & $0.15 \pm 0.13$ & $-0.01 \pm 0.04$ & $0.16 \pm 0.08$ \\
\hline Mel71 & $-0.09 \pm 0.01$ & $0.07 \pm 0.02$ & $0.04 \pm 0.04$ & $0.17 \pm 0.05$ & $-0.01 \pm 0.03$ & $-0.09 \pm 0.05$ \\
\hline Be81 & $0.23 \pm 0.05$ & $0.30 \pm 0.11$ & $0.19 \pm 0.12$ & $0.01 \pm 0.08$ & $0.24 \pm 0.09$ & $0.21 \pm 0.08$ \\
\hline NGC 6802 & $0.16 \pm 0.02$ & $0.34 \pm 0.06$ & $0.17 \pm 0.07$ & $0.16 \pm 0.05$ & $0.13 \pm 0.06$ & $0.11 \pm 0.02$ \\
\hline Rup134 & $0.16 \pm 0.02$ & $0.32 \pm 0.08$ & $0.15 \pm 0.08$ & $0.04 \pm 0.05$ & $0.17 \pm 0.05$ & $0.22 \pm 0.04$ \\
\hline NGC 6005 & $0.12 \pm 0.01$ & $0.24 \pm 0.03$ & $0.13 \pm 0.01$ & $0.08 \pm 0.06$ & $0.03 \pm 0.04$ & $0.12 \pm 0.04$ \\
\hline Pis 18 & $0.06 \pm 0.10$ & $0.25 \pm 0.08$ & $0.05 \pm 0.03$ & $0.14 \pm 0.08$ & $0.09 \pm 0.10$ & $0.16 \pm 0.02$ \\
\hline $\operatorname{Tr} 20$ & $0.13 \pm 0.03$ & $0.25 \pm 0.05$ & $0.13 \pm 0.09$ & $0.12 \pm 0.07$ & $0.10 \pm 0.05$ & $0.13 \pm 0.07$ \\
\hline $\mathrm{Be} 44$ & $0.34 \pm 0.05$ & - & - & $0.05 \pm 0.18$ & $0.23 \pm 0.07$ & $0.18 \pm 0.07$ \\
\hline NGC 2420 & $-0.14 \pm 0.04$ & $0.01 \pm 0.06$ & $0.04 \pm 0.05$ & $0.04 \pm 0.09$ & $-0.01 \pm 0.04$ & $-0.06 \pm 0.08$ \\
\hline Be31 & $-0.32 \pm 0.05$ & $-0.11 \pm 0.08$ & $-0.09 \pm 0.09$ & $-0.32 \pm 0.02$ & $-0.14 \pm 0.07$ & $-0.16 \pm 0.04$ \\
\hline $\mathrm{Be} 25$ & $-0.24 \pm 0.13$ & $0.17 \pm 0.21$ & $0.01 \pm 0.22$ & $-0.28 \pm 0.23$ & $0.03 \pm 0.11$ & $0.00 \pm 0.09$ \\
\hline NGC 2243 & $-0.41 \pm 0.08$ & $-0.21 \pm 0.06$ & $-0.34 \pm 0.07$ & - & $-0.34 \pm 0.08$ & $-0.31 \pm 0.10$ \\
\hline M67 & $-0.07 \pm 0.02$ & $0.08 \pm 0.08$ & $0.01 \pm 0.03$ & $0.00 \pm 0.09$ & $0.16 \pm 0.19$ & $-0.10 \pm 0.09$ \\
\hline $\mathrm{Be} 36$ & $-0.18 \pm 0.04$ & $-0.10 \pm 0.04$ & $-0.11 \pm 0.05$ & - & $-0.10 \pm 0.20$ & $-0.01 \pm 0.07$ \\
\hline
\end{tabular}

velocity are consistent with those of the cluster, we can make several hypotheses on the nature of this star: (i) it is not a cluster member, but a field star with exactly the same metallicity and radial velocity as the cluster; (ii) it is a remnant of a binary system in which one of two companions was merged thus modifying the surface composition of the other companion; (iii) the different composition is due to planet engulfment (see, e.g. Spina et al. 2015). However, the study of this peculiar star is beyond the scope of the present paper and deserves further investigation.

\subsection{The origin of the s-process elements}

Most of the neutron capture elements are produced in a mixture of astrophysical environments, involving both the $r$ - and $s$-processes. There are a few cases in which one of the two processes dominates over the other, being then the major process responsible for the element production, but in general both processes contribute, with one or the other dominating at different epochs and thus in different metallicity ranges. 
Table 4. Abundance ratios in open clusters, expressed in the form $[\mathrm{X} / \mathrm{Fe}]$.

\begin{tabular}{|c|c|c|c|c|c|c|}
\hline Id & [YII/Fe] & {$[\mathrm{ZrII} / \mathrm{Fe}]$} & {$[\mathrm{CeII} / \mathrm{Fe}]$} & {$[\mathrm{BaII} / \mathrm{Fe}]$} & [LaII/Fe] & [EuII/Fe] \\
\hline NGC 6067 & $0.14 \pm 0.09$ & $0.20 \pm 0.10$ & $0.07 \pm 0.10$ & $0.39 \pm 0.11$ & $0.13 \pm 0.09$ & $0.16 \pm 0.10$ \\
\hline NGC 2516 & $0.11 \pm 0.06$ & $0.58 \pm 0.07$ & $0.38 \pm 0.10$ & $0.20 \pm 0.08$ & - & - \\
\hline NGC 6259 & $-0.01 \pm 0.05$ & $0.13 \pm 0.08$ & $-0.06 \pm 0.12$ & $-0.04 \pm 0.06$ & $-0.01 \pm 0.11$ & $-0.05 \pm 0.06$ \\
\hline NGC 3532 & $0.06 \pm 0.13$ & - & - & $0.17 \pm 0.11$ & - & - \\
\hline NGC 6705 & $0.03 \pm 0.06$ & $0.15 \pm 0.08$ & $-0.01 \pm 0.10$ & $0.15 \pm 0.12$ & $0.03 \pm 0.08$ & $0.01 \pm 0.08$ \\
\hline NGC 6633 & $0.11 \pm 0.10$ & $0.24 \pm 0.09$ & $0.18 \pm 0.09$ & $0.24 \pm 0.14$ & $0.15 \pm 0.10$ & $-0.05 \pm 0.16$ \\
\hline NGC 4815 & $0.11 \pm 0.12$ & $0.29 \pm 0.14$ & $0.13 \pm 0.12$ & $0.31 \pm 0.13$ & $0.12 \pm 0.12$ & $-0.05 \pm 0.13$ \\
\hline $\operatorname{Tr} 23$ & $0.01 \pm 0.05$ & $0.22 \pm 0.05$ & $-0.01 \pm 0.11$ & $0.05 \pm 0.14$ & $-0.03 \pm 0.05$ & $0.00 \pm 0.09$ \\
\hline Mel71 & $0.08 \pm 0.02$ & $0.23 \pm 0.02$ & $0.21 \pm 0.04$ & $0.34 \pm 0.05$ & $0.16 \pm 0.03$ & $-0.05 \pm 0.06$ \\
\hline Be81 & $0.10 \pm 0.08$ & $0.19 \pm 0.12$ & $0.05 \pm 0.14$ & $-0.10 \pm 0.10$ & $0.11 \pm 0.10$ & $0.00 \pm 0.10$ \\
\hline NGC 6802 & $0.15 \pm 0.06$ & $0.36 \pm 0.08$ & $0.18 \pm 0.09$ & $0.16 \pm 0.07$ & $0.15 \pm 0.08$ & $-0.01 \pm 0.06$ \\
\hline Rup134 & $-0.04 \pm 0.06$ & $0.14 \pm 0.09$ & $-0.02 \pm 0.10$ & $-0.15 \pm 0.08$ & $-0.06 \pm 0.08$ & $-0.08 \pm 0.07$ \\
\hline NGC 6005 & $-0.01 \pm 0.01$ & $0.12 \pm 0.03$ & $0.03 \pm 0.01$ & $-0.03 \pm 0.05$ & $-0.07 \pm 0.04$ & $-0.14 \pm 0.04$ \\
\hline Pis 18 & $0.06 \pm 0.10$ & $0.20 \pm 0.07$ & $0.04 \pm 0.03$ & $0.08 \pm 0.08$ & $0.06 \pm 0.10$ & $0.00 \pm 0.02$ \\
\hline $\operatorname{Tr} 20$ & $0.08 \pm 0.07$ & $0.20 \pm 0.08$ & $0.10 \pm 0.11$ & $0.08 \pm 0.09$ & $0.04 \pm 0.08$ & $-0.03 \pm 0.09$ \\
\hline $\mathrm{Be} 44$ & $0.13 \pm 0.07$ & - & $0.06 \pm 0.10$ & - & $0.05 \pm 0.09$ & $-0.12 \pm 0.09$ \\
\hline NGC 2420 & $0.07 \pm 0.05$ & $0.22 \pm 0.07$ & $0.21 \pm 0.06$ & $0.23 \pm 0.09$ & $0.18 \pm 0.05$ & $0.00 \pm 0.08$ \\
\hline $\mathrm{Be} 31$ & $0.05 \pm 0.05$ & $0.28 \pm 0.09$ & $0.31 \pm 0.10$ & $0.06 \pm 0.04$ & $0.23 \pm 0.08$ & $0.09 \pm 0.06$ \\
\hline $\mathrm{Be} 25$ & $0.11 \pm 0.22$ & $0.30 \pm 0.27$ & $0.34 \pm 0.28$ & $0.05 \pm 0.29$ & $0.21 \pm 0.20$ & $0.19 \pm 0.19$ \\
\hline NGC 2243 & $0.07 \pm 0.09$ & $0.28 \pm 0.08$ & $0.16 \pm 0.09$ & - & $0.19 \pm 0.10$ & $0.12 \pm 0.12$ \\
\hline M67 & $-0.01 \pm 0.04$ & $0.14 \pm 0.08$ & $0.05 \pm 0.05$ & $0.08 \pm 0.10$ & $0.24 \pm 0.19$ & $-0.19 \pm 0.10$ \\
\hline Be36 & $0.05 \pm 0.20$ & $0.18 \pm 0.20$ & $0.10 \pm 0.20$ & - & $0.11 \pm 0.27$ & $0.13 \pm 0.20$ \\
\hline
\end{tabular}

Table 5. Solar percentages and isotopic composition for the $s$-process elements from Sneden et al. (2008) and Bisterzo et al. (2014).

\begin{tabular}{llll}
\hline \hline Element & \multicolumn{1}{c}{ Isotopes } & Sneden et al. (2008)(\%) & Bisterzo et al. (2014)(\%) \\
\hline $\mathrm{Y}$ & ${ }^{89} \mathrm{Y}$ & 72 & 72 \\
$\mathrm{Zr}$ & ${ }^{90} \mathrm{Zr},{ }^{91} \mathrm{Zr},{ }^{92} \mathrm{Zr},{ }^{94} \mathrm{Zr},{ }^{96} \mathrm{Zr}$ & $52,11,15,17,3(81)$ & 66 \\
$\mathrm{La}$ & ${ }^{139} \mathrm{La}$ & 75 & 76 \\
$\mathrm{Ce}$ & ${ }^{140} \mathrm{Ce},{ }^{142} \mathrm{Ce}$ & $90,10(81)$ & 84 \\
$\mathrm{Ba}$ & ${ }^{134} \mathrm{Ba},{ }^{135} \mathrm{Ba},{ }^{136} \mathrm{Ba},{ }^{137} \mathrm{Ba},{ }^{138} \mathrm{Ba}$ & $3,7,9,12,69(85)$ & 85 \\
\hline
\end{tabular}

In Figs. 3 and 4, we show the abundance ratios $[\mathrm{X} / \mathrm{Fe}]$ of the $s$-process elements ordered by increasing atomic number plotted with respect to $[\mathrm{Fe} / \mathrm{H}]$. Figure 3 presents the thin and thick individual stars' abundances and the open cluster median abundances: this figure allows us to estimate the differences between field stars and open clusters. We note that the age and distance distributions of the cluster and field star samples plotted here differ in important ways. The majority of field stars have ages between 3 and $7 \mathrm{Gyr}$, with very few stars being younger than 2 Gyr. By contrast, all but six of the open clusters are younger than 2 Gyr. In addition, the fields stars have been limited to $1.5 \mathrm{kpc}$ around the solar radius, in the range $6.5 \mathrm{kpc}<R_{\mathrm{GC}}<9.5 \mathrm{kpc}$, while the open clusters range from 4.5 to $18 \mathrm{kpc}$ in Galactocentric radius. Consequently, any age- and distance-dependent trends in abundances will affect the cluster and field star distributions in these diagrams differently. To help understand the impact of the distance differences, we have colour-coded the open clusters according to their Galactocentric distance, separating the clusters into radial ranges of $R_{\mathrm{GC}}<6.5 \mathrm{kpc}$ (blue), $6.5 \mathrm{kpc}<R_{\mathrm{GC}}<9.5 \mathrm{kpc} \quad$ (green), and $R_{\mathrm{GC}}>9.5 \mathrm{kpc}$ (violet).

Figure 4 shows the thin and thick abundances, averaged in bins of 0.1 dex in width: this figure aims at comparing the different Galactic populations and seeking differences between thickand thin-disc stars. The binned results are only shown in the figures if there is more than one star in the considered interval. The errors on the binned values are $1-\sigma$ standard deviations of the average. Typical errors on the individual abundances are: $0.15 \mathrm{dex}$ for $\log (\mathrm{Y} \mathrm{II} / \mathrm{H}), 0.15 \mathrm{dex}$ for $\log (\mathrm{Zr} \mathrm{II} / \mathrm{H}), 0.08 \mathrm{dex}$ for $\log (\mathrm{Ba} \mathrm{II} / \mathrm{H}), 0.09 \mathrm{dex}$ for $\log (\mathrm{La} \mathrm{II} / \mathrm{H}), 0.14 \operatorname{dex}$ for $\log (\mathrm{Ce} \mathrm{II} / \mathrm{H})$, and 0.16 dex for $\log (\mathrm{Eu} \mathrm{II} / \mathrm{H})$.

In the first panel of both Figs. 3 and 4 , we present the $[\alpha / \mathrm{Fe}]$ versus $[\mathrm{Fe} / \mathrm{H}]$ distribution of field stars, with the chemical dividing line between thin- and thick-disc stars. To separate thin and thick stars we have used the relationship of Adibekyan et al. (2011). As expected from their young ages, most open clusters belong to the thin-disc populations. The separation between the thin- and thick-disc stars is clear and in agreement with previous results (e.g. Recio-Blanco et al. 2014).

In the second panels, $[\mathrm{Y} / \mathrm{Fe}]$ is almost flat across the whole metallicity range, with a relatively small dispersion and a slight decrease towards the lower metallicity. The cluster abundances occupy the upper envelope of the region defined by the abundances of field stars, while the distributions of thin- and thickdisc stars are coincident (second panel of Fig. 3). This difference between cluster and field stars is seen here for the first time with a statistically significant sample of cluster members and of field stars. In the following sections, we show that this is likely an evolutionary effect due to the younger ages of cluster stars with respect to the field population. 


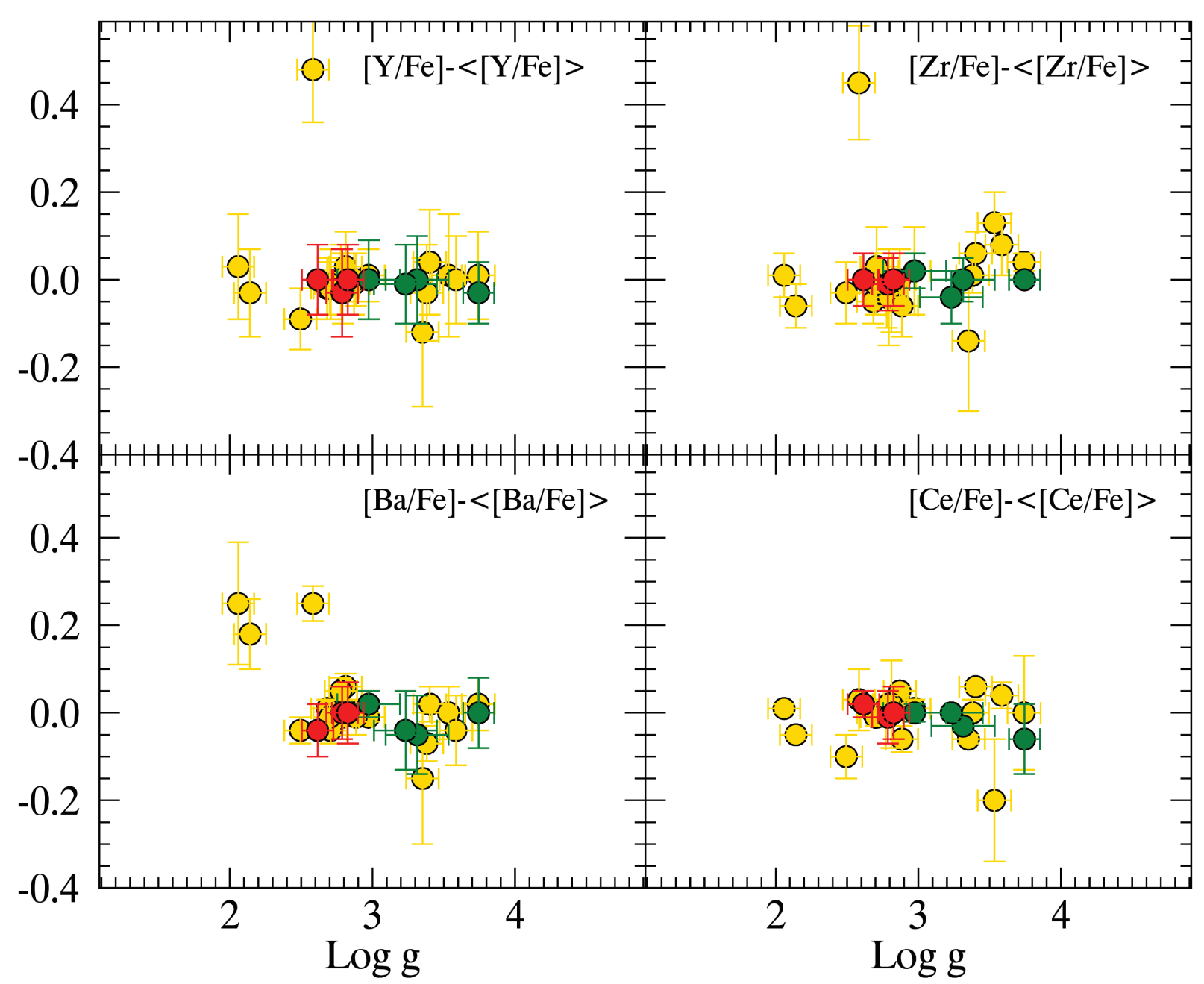

Fig. 1. Abundance ratios $[\mathrm{X} / \mathrm{Fe}]$ normalised to the median abundance of the cluster vs. logg in member stars of the open clusters NGC 2420 (yellow), NGC 6633 (red), and M67 (green). Stars with errors in the abundances larger than 0.2 dex are not included in the plot.

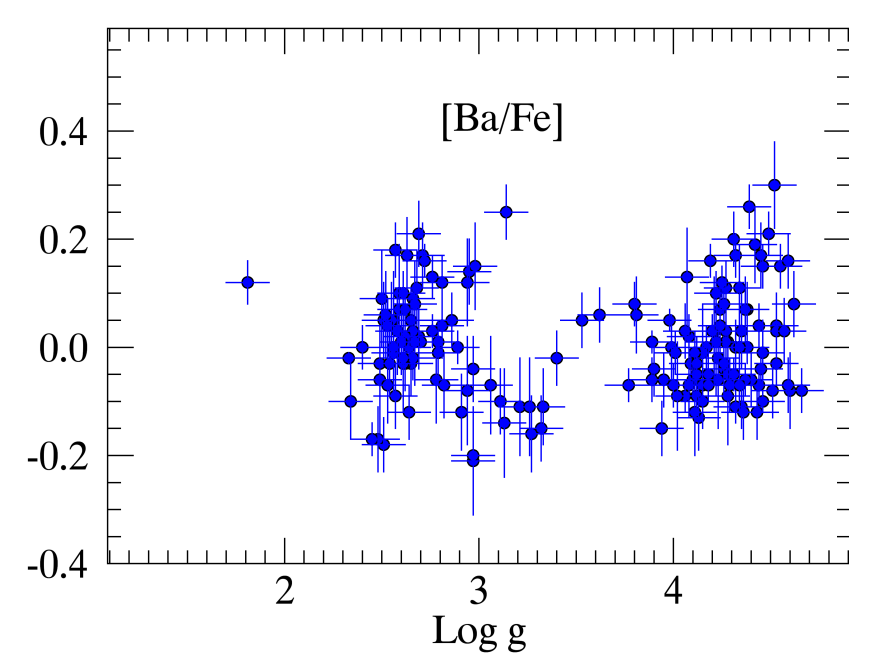

Fig. 2. Abundance ratios $[\mathrm{Ba} / \mathrm{Fe}]$ vs. $\log g$ in Milky Way field stars with $-0.1<[\mathrm{Fe} / \mathrm{H}]<0.1$.

In the third panels, $[\mathrm{Zr} / \mathrm{Fe}]$ increases both in thin- and thickdisc stars towards lower metallicity, indicating a non-negligible contribution from massive stars, which, given its higher $s$-process percentage with respect to $\mathrm{Y}$, is slightly unexpected. The recent paper by Delgado Mena et al. (2017) also indicates that $\mathrm{Zr}$ shows a clear increasing trend for lower metallicities. The trend is not new, and has been observed in previous works (e.g. González Hernández et al. 2010; Mishenina et al. 2013; Battistini \& Bensby 2016; Zhao et al. 2016). The behaviour of $\mathrm{Zr}$ can be related to a reassessment of its $s$-process contribution. The work of Bisterzo et al. (2014) indicates that only $\sim 66 \%$ of $\mathrm{Zr}$ is produced by the $s$-process in the Sun. Other processes may contribute to the production of $\mathrm{Zr}$, as discussed by Travaglio et al. (2004) who introduced the LEPP process associated with massive stars to explain the abundance patterns of the neutron capture elements, especially at low metallicity. As in the case of $[\mathrm{Y} / \mathrm{Fe}],[\mathrm{Zr} / \mathrm{Fe}]$ in open clusters is slightly higher with respect to thin and thick stars, which appear statistically indistinguishable, although both clusters and field stars have $[\mathrm{Zr} / \mathrm{Fe}]$ elevated above the solar ratio.

With a percentage of $85 \%, \mathrm{Ba}$ (fourth panels of Figs. 3 and 4 ) is dominated by the $s$-process in the metallicity range from $[\mathrm{Fe} / \mathrm{H}] \geq-1.0$ to super-solar metallicity. Only at lower metallicities, does the primary $r$-process contribution, relatively small at the time of formation of the Sun, play an important role (Travaglio et al. 1999). In the field stars, the abundance ratio $[\mathrm{Ba} / \mathrm{Fe}]$ is close to solar for metallicities between $[\mathrm{Fe} / \mathrm{H}]=-1.0$ and 0 , with a peak of $[\mathrm{Ba} / \mathrm{Fe}] \sim 0.1 \mathrm{dex}$ in the range $-0.4<[\mathrm{Fe} / \mathrm{H}]<-0.6$. In the super-solar metallicity range, we distinguish between $[\mathrm{Ba} / \mathrm{Fe}]$ in open clusters and field stars; $[\mathrm{Ba} / \mathrm{Fe}] \geq 0$ dex for clusters located in the solar neighbour- 
hood and negative for the innermost, more metal-rich clusters and for the thin- and thick-disc stars. The thick-disc abundance ratios are systematically lower than the thin-disc ratios, with the exception of stars with $[\mathrm{Fe} / \mathrm{H}]>0$ dex for which thin- and thickdisc stars have coincident $[\mathrm{Ba} / \mathrm{Fe}]$ distributions.

Among the second-peak elements, La (fifth panels of Figs. 3 and 4) has a slight increase of [La/Fe] towards lower metallicity. The open clusters are located in the upper envelope of the distribution of field star abundance ratios. Thin- and thick-disc stars show similar patterns, with thin-disc stars slightly overabundant in $[\mathrm{La} / \mathrm{Fe}]$.

Finally, the behaviour of $[\mathrm{Ce} / \mathrm{Fe}]$ (sixth panels of Figs. 3 and 4$)$ is almost flat in the $[\mathrm{Fe} / \mathrm{H}]$ range considered. At variance with $[\mathrm{Ba} / \mathrm{Fe}],[\mathrm{Ce} / \mathrm{Fe}]$ is also constant at super-solar metallicities. Thin- and thick-disc stars share similar patterns. For both elements $\mathrm{Ce}$ and $\mathrm{La}$, the more metal-poor open clusters have higher $[\mathrm{La} / \mathrm{Fe}]$ and $[\mathrm{Ce} / \mathrm{Fe}]$ than the more metal-rich ones. However, these metal-poor clusters are located in the outer disc and their abundances may be more a reflection of the radial gradient of these elements in the Galactic disc.

\subsection{Estimating the r-process contribution}

The abundance ratio $[\mathrm{X} / \mathrm{Eu}]$ can be used directly to judge the relative importance of the $r$-process channels during the evolution of the Galaxy in the metallicity range of our sample stars, as has been done in previous works (e.g. Mashonkina \& Gehren 2001; Battistini \& Bensby 2016). In Figs. 5 and 6, we present the $[\mathrm{X} / \mathrm{Eu}]$ abundance ratios versus $[\mathrm{Fe} / \mathrm{H}]$. In the plots, the blue dashed lines represent the $s$-process solar values, while the red dot-dashed line indicates the pure $r$-process contribution of both elements, derived using the percentages of Bisterzo et al. (2014) and the solar abundances of Grevesse et al. (2007). The two figures highlight different aspects: in the former we have a direct comparison between field stars and open clusters, and in the latter the comparison between thin- and thick-disc populations.

In the first panels of both figures, we present the abundance ratio $[\mathrm{Eu} / \mathrm{Fe}]$ versus $[\mathrm{Fe} / \mathrm{H}]$. Europium is an $r$-process element and shows a clear increase towards the lower metallicity. Although the first hypothesis about the mechanisms responsible of $r$-process nucleosynthesis date back to many years ago (Burbidge et al. 1957; Seeger et al. 1965), there is still an open debate about the dominant production site of the $r$-process elements (see e.g. Thielemann et al. 2011). The $r$-process nucleosynthesis must be associated with both the evolution of massive stars $\left(M>10 M_{\odot}\right)$ and with neutron star mergers (Matteucci et al. 2014). Very recently, observations of the optical counterpart of a gravitational wave source have identified the presence of $r$-process elements in the spectrum of the merger of two neutron stars, clearly pointing to the production of these elements in neutron-star merger events (Côté et al. 2018).

In the second panels, for the first-peak element Y, the sample shows an increasing contribution from $s$-process elements above a pure $r$-process level, but there are some stars in the range $-1.0<[\mathrm{Fe} / \mathrm{H}]<-0.5$ that appear to have a pure $r$-process composition. These stars belong to both the thin and thick disc populations. Over the full metallicity range, the abundance ratios of the thick- and thin-disc stars are offset from each other by about 0.1 dex in $[\mathrm{Y} / \mathrm{Eu}]$.

In the third panels, we present the abundance ratio $[\mathrm{Zr} / \mathrm{Eu}]$ versus $[\mathrm{Fe} / \mathrm{H}]$, which is almost constant, quite far from the pure $r$-process contribution, and slightly above the solar value. Almost all the open clusters have positive [Zr/Eu]. For this element, the thin- and thick-disc populations are separated: the abundance ratios of thick-disc stars are lower, although they follow a similar pattern to the thin-disc stars (see third panel of Fig. 6). In the fourth panels, barium shows a mild increase of the $r$-process component towards low metallicity. In the thin-disc population, $[\mathrm{Ba} / \mathrm{Eu}]$ is close to solar values from $[\mathrm{Fe} / \mathrm{H}] \sim+0.4$ down to $[\mathrm{Fe} / \mathrm{H}] \sim-0.5 \mathrm{dex}$, and subsequently decreases. For the thick-disc stars, the decline starts at higher metallicity, $[\mathrm{Fe} / \mathrm{H}] \sim-0.2 \mathrm{dex}$, and again, over the entire metallicity range, the thick- disc stars have lower abundance ratios. $[\mathrm{Ba} / \mathrm{Eu}]$ in open clusters is positive in the range $-0.2<[\mathrm{Fe} / \mathrm{H}]<+0.2 \mathrm{dex}$, while it is close to zero for the clusters more metal-poor and more metal-rich.

In the fifth panels, $[\mathrm{La} / \mathrm{Eu}]$ is quite flat over the whole metallicity range. The open clusters are all slightly over solar in $[\mathrm{La} / \mathrm{Eu}]$, and thick disc abundance ratios are slightly lower than in thin- disc stars.

In the sixth panels, $[\mathrm{Ce} / \mathrm{Eu}]$ is also quite flat over the whole metallicity range and it tends to increase in both thin and thickdisc stars towards high $[\mathrm{Fe} / \mathrm{H}]$. The few stars at low metallicity $[\mathrm{Fe} / \mathrm{H}] \leq-0.5$ dex might point to a decrease of $[\mathrm{Ce} / \mathrm{Eu}]$, indicating the beginning of the $r$-process contribution in the thick-disc stars.

We can therefore conclude that in the considered metallicity range, the production of the five elements is clearly dominated by the $s$-process. At any metallicity, the thick- disc stars show very slightly lower $[\mathrm{s} / \mathrm{Eu}]$ ratios than the thin- disc population, consistent with their slightly higher $[\mathrm{Eu} / \mathrm{Fe}]$ values.

\section{The time evolution of the abundances of the $s$-process elements}

One of the most challenging issues in the field of chemical evolution is the nature of the time-evolution of the $s$-process abundances. The classical chemical and chemo-dynamical models (Pagel \& Tautvaisiene 1997; Travaglio et al. 1999, 2004; Raiteri et al. 1999), based on the accepted scenario for $s$-processing at that time, predicted a plateau or even a decrease in the abundances $[\mathrm{X} / \mathrm{Fe}]$ versus age after the solar formation. A late increase was not expected. However, recently, after the original work of D'Orazi et al. (2009), a number of works have claimed the presence of a continuous increase of the first- and second-peak element abundances, sometimes with a further growth starting 5-6 Gyr ago (Maiorca et al. 2011; da Silva et al. 2012; Nissen 2016; Spina et al. 2016; Nissen et al. 2017; Silva Aguirre et al. 2017; Feltzing et al. 2017).

In the present work, we combine the open cluster sample, which basically maps the recent epochs in the evolution of the Galactic disc, with the field stars, divided into thin- and thick-disc populations on the basis of their $[\alpha / \mathrm{Fe}]$, as explained in Sect. 4.1. The ages of the field stars, computed with the isochrone projection methods, are much less accurate than the ages of open clusters, and they have only a statistical meaning. However, keeping in mind the limit of measuring stellar ages, the Gaia-ESO IDR5 sample gives us the opportunity, for the first time, to combine the largest sample of $s$-process element abundances measured in a completely homogeneous way in very young and very old stars. No biases due to, for instance, the line list selection, the method of deriving abundances, or adopted reference abundances are present in the Gaia-ESO IDR5.

In Figs. 7-9, we show the abundance ratios $[\mathrm{X} / \mathrm{Fe}]$ versus age of both field stars and open clusters. We reiterate that the sample of thin- and thick- disc stars is already restricted to the solar neighbourhood, that is, $6.5 \mathrm{kpc}<R_{\mathrm{GC}}<9.5 \mathrm{kpc}$. The open clusters span a much larger range of distances, and we have 


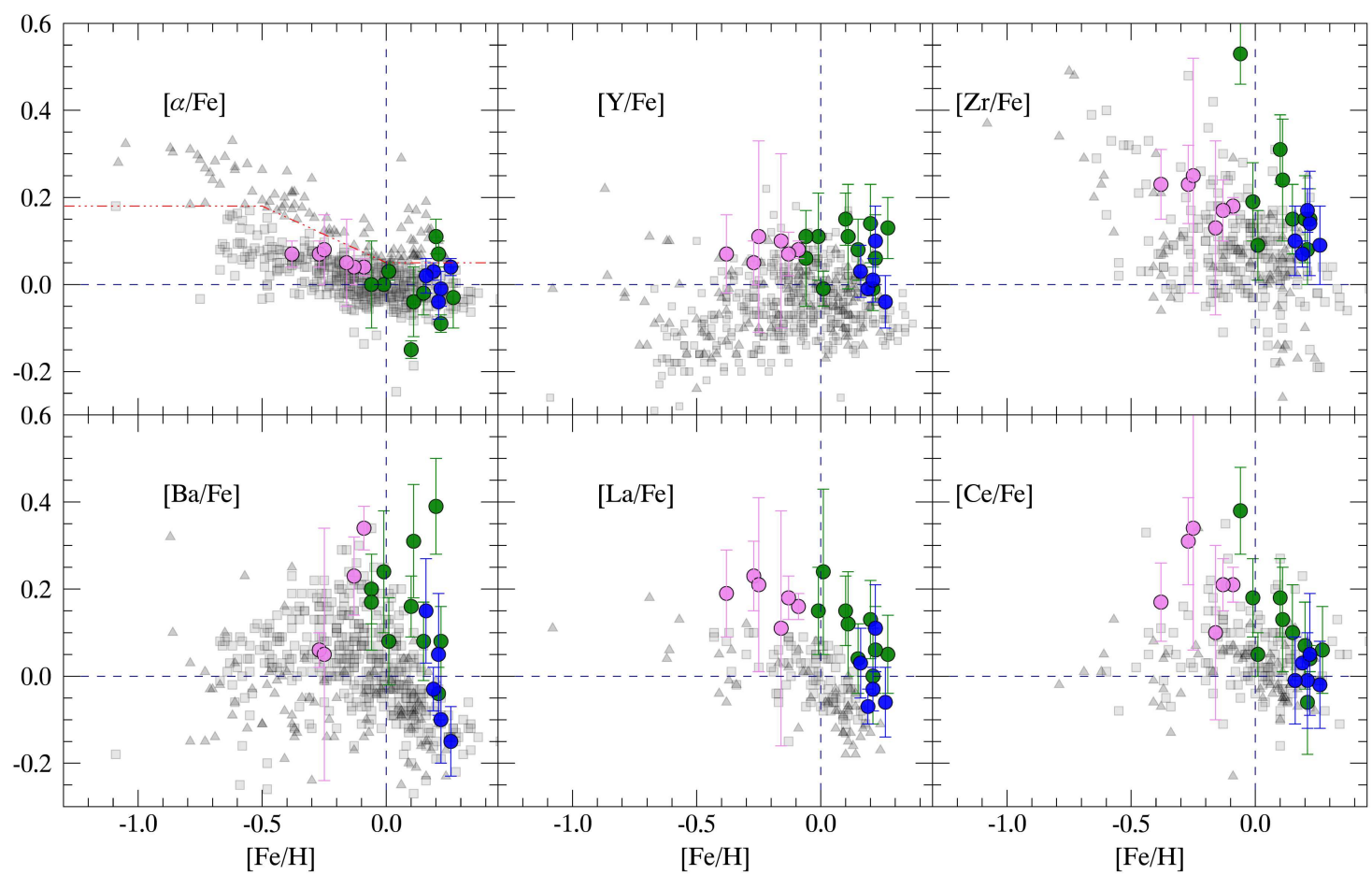

Fig. 3. Abundance ratios $[\mathrm{X} / \mathrm{Fe}]$ vs. $[\mathrm{Fe} / \mathrm{H}]$ in member stars of clusters (large filled circles - in blue for clusters with $R_{\mathrm{GC}}<6.5 \mathrm{kpc}$, in green for clusters with $6.5 \mathrm{kpc}<R_{\mathrm{GC}}<9.5 \mathrm{kpc}$, and in violet for clusters with $R_{\mathrm{GC}}>9.5 \mathrm{kpc}$ ), in thin-disc stars (small grey squares) and in thick-disc stars (small black triangles). The blue dashed lines represent the solar values. The red dash-dotted line is the relationship of Adibekyan et al. (2011) which separates thin- and thick-disc stars.

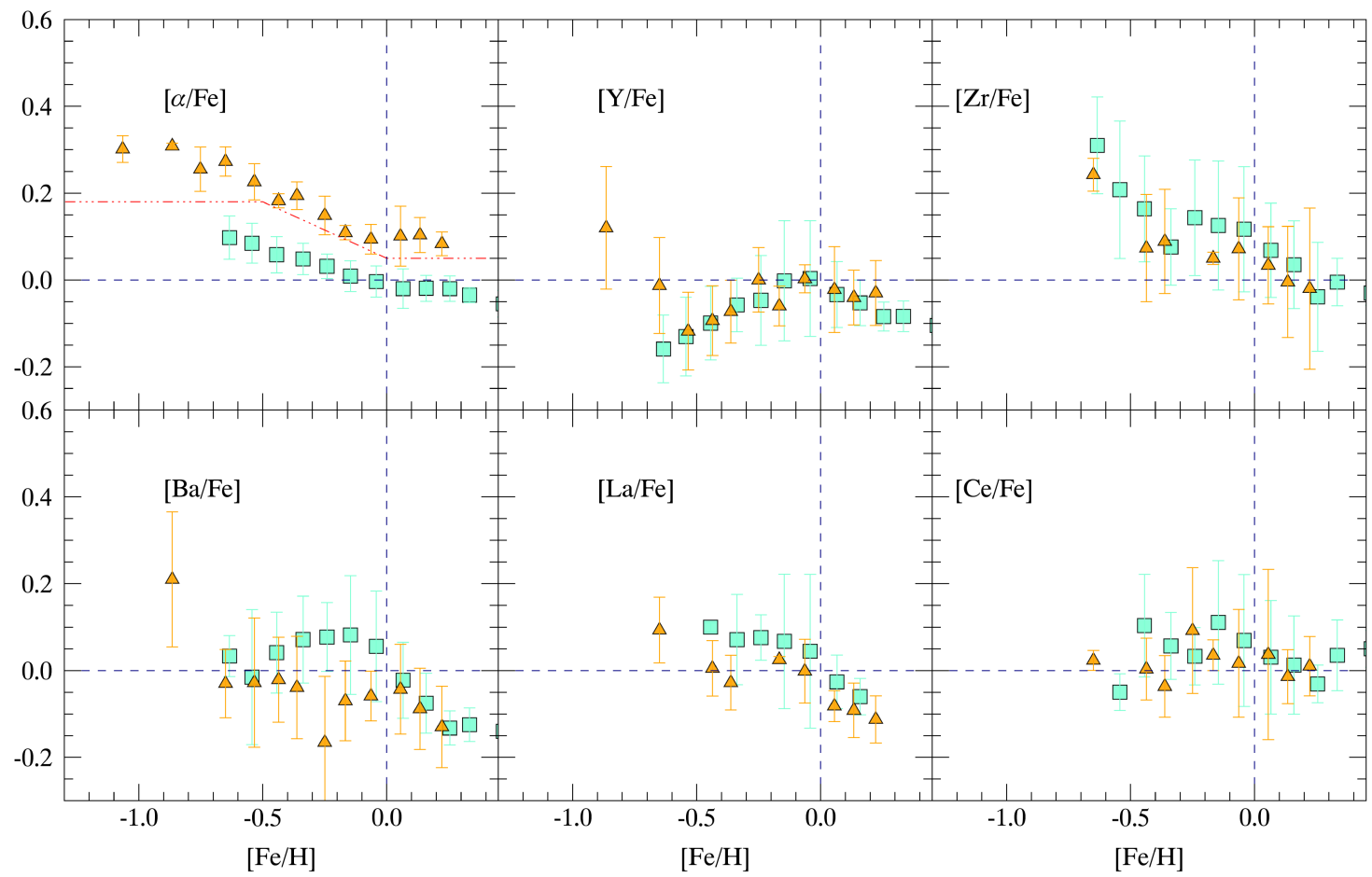

Fig. 4. Abundance ratios $[\mathrm{X} / \mathrm{Fe}]$ vs. $[\mathrm{Fe} / \mathrm{H}]$ in thin-disc stars (cyan squares, binned results, bin width 0.1 dex) and in thick-disc stars (orange triangles, binned results, bin width 0.1 dex). The blue dashed lines represent the solar values. The red dash-dotted line is the relationship of Adibekyan et al. (2011) which separates thin- and thick-disc stars.

distinguished them by radial bins according to their Galactocentric location, denoting inner-disc, solar-neighbourhood, and outer-disc clusters separately in the figures. In Fig. 7, we compare the field stars with the open clusters, while in Fig. 8, we compare the thin and thick disc $[\mathrm{X} / \mathrm{Fe}]$ versus stellar ages (bin- ning in age bins 1 Gyr wide). Finally, in Fig. 9, we limit our analysis to the solar-neighbourhood thin-disc populations, and open clusters belonging to the same radial range.

Figure 7 aims at highlighting the behaviour of clusters located at different Galactocentric distance with respect to the field 


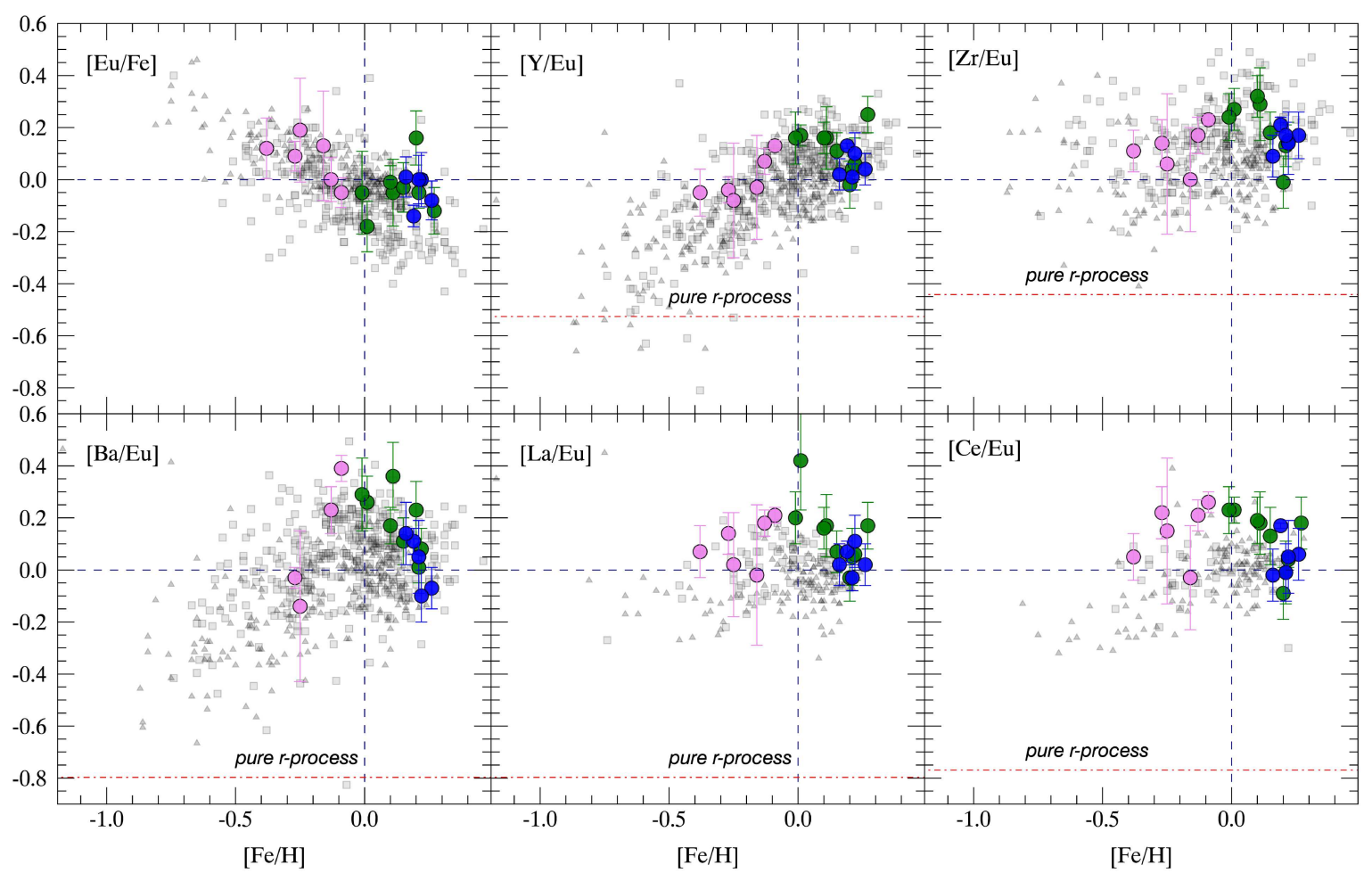

Fig. 5. $[\mathrm{Eu} / \mathrm{Fe}]$ and abundance ratios $[\mathrm{X} / \mathrm{Eu}]$ vs. $[\mathrm{Fe} / \mathrm{H}]$. Symbols and colour codes are as in Fig. 3. The blue dashed lines represent the solar values, while the red dot-dashed lines indicate the abundance ratio below which the pure $r$-contribution dominates, derived using the percentages of Bisterzo et al. (2014) and the solar abundances of Grevesse et al. (2007).

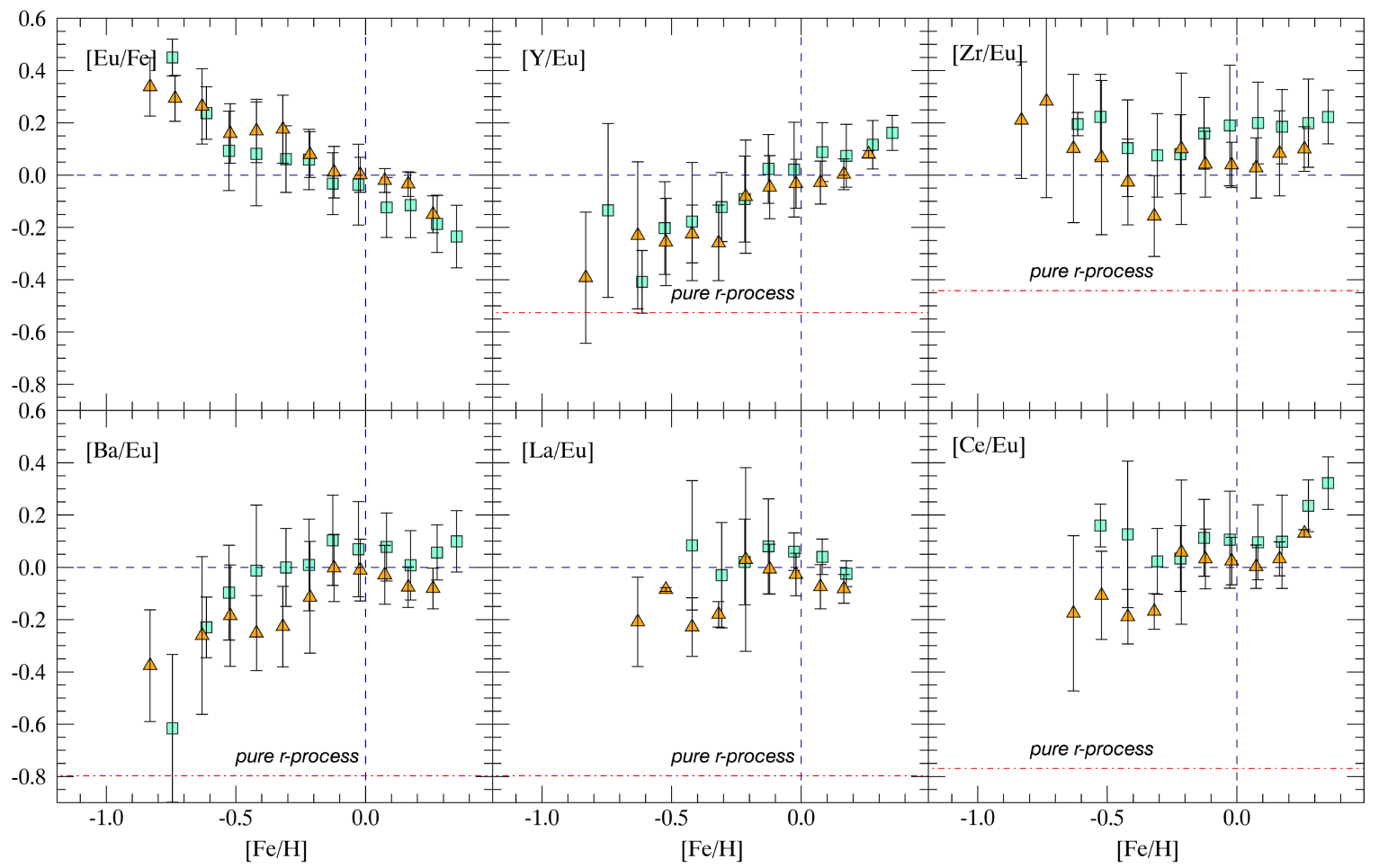

Fig. 6. $[\mathrm{Eu} / \mathrm{Fe}]$ and abundance ratios $[\mathrm{X} / \mathrm{Eu}]$ vs. $[\mathrm{Fe} / \mathrm{H}]$. Symbols and colour codes are as in Fig. 4. The lines are coded as in Fig. 5.

stars. The solar-neighbourhood and inner-disc clusters populate the youngest age regions, while the outer-disc clusters span a large range of ages. For all $s$-process elements, we notice a difference between the youngest clusters of the solar neighbourhood and the inner-disc clusters. The former usually have higher $[\mathrm{X} / \mathrm{Fe}]$ than the latter. The outer-disc open clusters typically have $[\mathrm{X} / \mathrm{Fe}]$ sim- ilar to the solar-neighbourhood clusters. Dividing the cluster sample by Galactocentric distance makes clear that there is a strong dependence of the heavy $s$-element abundances on the location of the clusters. The behaviour of $[\mathrm{X} / \mathrm{Fe}]$ with Galactocentric radius seems to be complex and likely related to different star formation histories and to metallicity dependency of the stellar yields. 


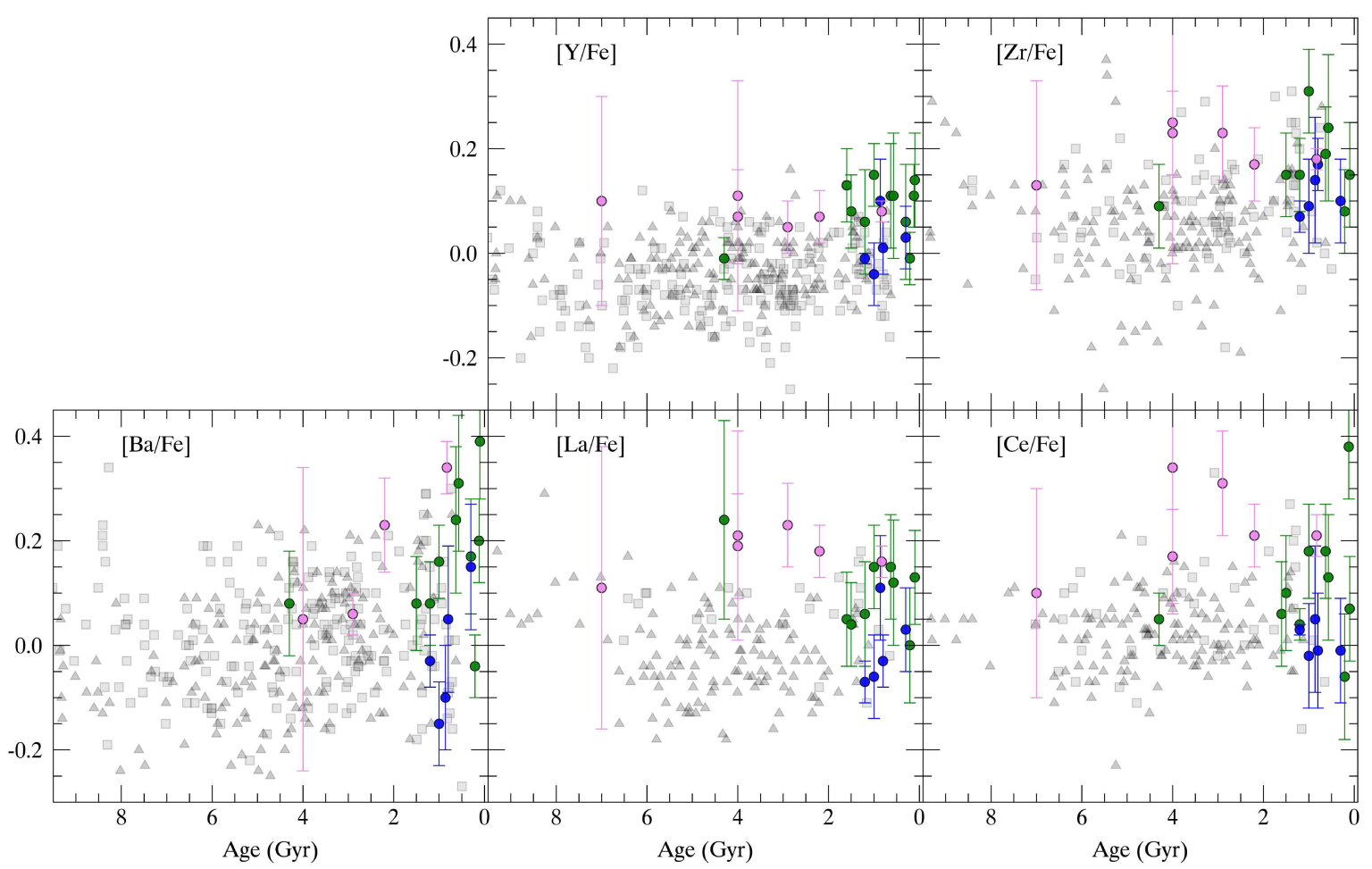

Fig. 7. Abundance ratios $[\mathrm{X} / \mathrm{Fe}]$ vs. Age. Symbol and colour codes as in Fig. 3.

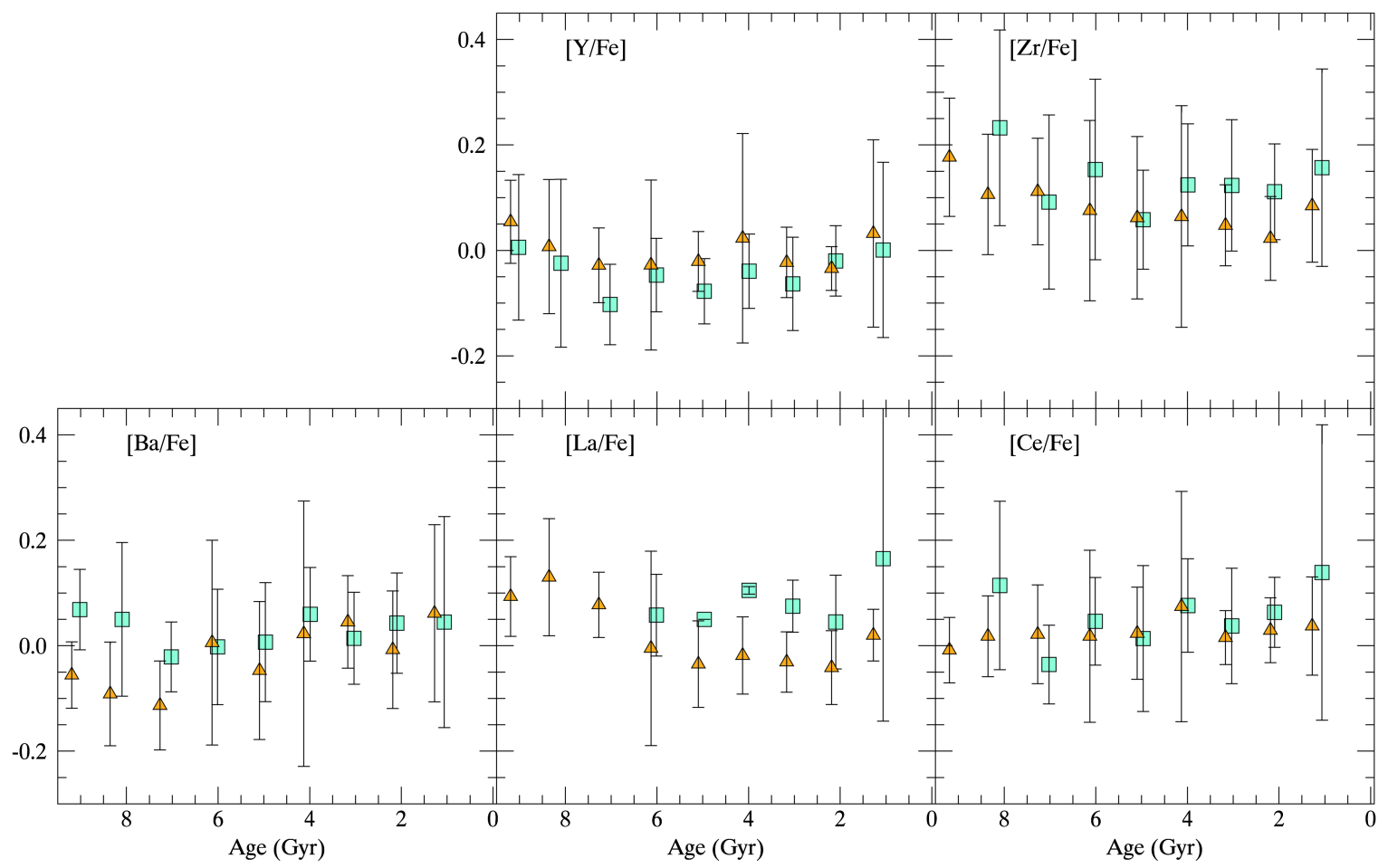

Fig. 8. Abundance ratios $[\mathrm{X} / \mathrm{Fe}]$ vs. Age. Symbol and colour codes as in Fig. 4.

Figure 8 shows $[\mathrm{X} / \mathrm{Fe}]$ as a function of stellar age for the binned thin- and thick-disc populations. In the radial range shown here, that is, $6.5 \mathrm{kpc}<R_{\mathrm{GC}}<9.5 \mathrm{kpc}$, there are not strong differences between the behaviours of the thin- and thick-disc populations. All elements seem to indicate a slight increase in [X/Fe] starting at ages $<8 \mathrm{Gyr}$. These trends are most clear in the thin disc, where the sample sizes per bin are larger and mean values are perhaps better determined than for the thick-disc stars.

Figure 9 presents $[\mathrm{X} / \mathrm{Fe}]$ versus stellar age for the thindisc and cluster populations in the solar neighbourhood with weighted linear fits to the combined cluster and thin-disc samples for ages lower than $8 \mathrm{Gyr}$, that is, corresponding to the epoch in which we expect the contribution of the $s$-process to 


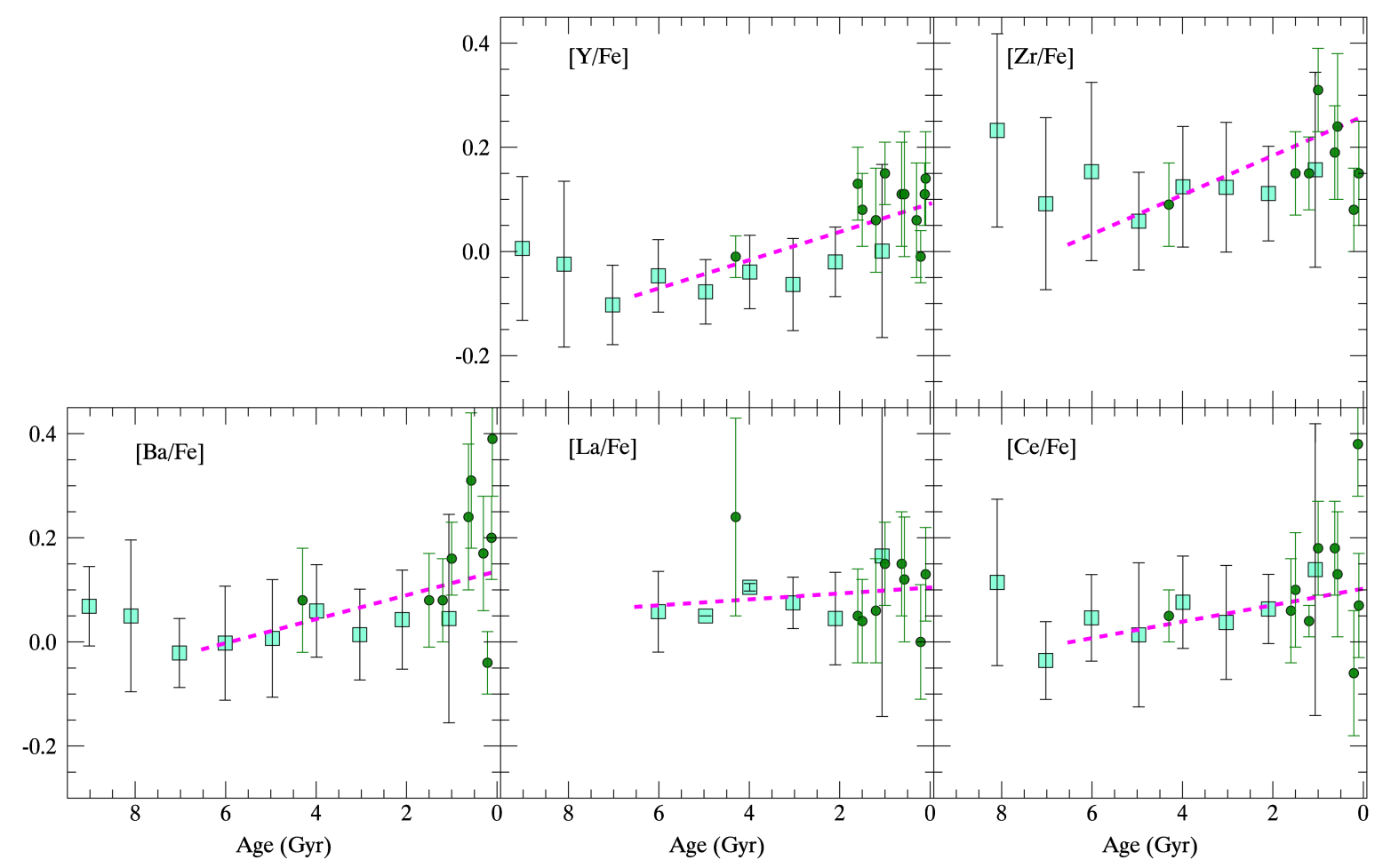

Fig. 9. Abundance ratios $[\mathrm{X} / \mathrm{Fe}]$ vs. Age for the thin-disc stars (binned results, bin 0.1 dex wide, cyan squares) and the open clusters located in the solar neighborhood (green circles). The magenta dashed lines are the weighted linear fits to the cumulative sample of solar neighbourhood clusters and thin-disc field stars (see Table 6 for the coefficients) for stellar ages $<8 \mathrm{Gyr}$.

begin. In Table 6, we present these weighted linear fits, reporting the slopes, the intercepts, and correlation coefficients for fits by element. Restricting our sample to the solar neighbourhood allows us to better appreciate the increasing trends with decreasing stellar ages. The first peak elements, $[\mathrm{Y} / \mathrm{Fe}]$ and $[\mathrm{Zr} / \mathrm{Fe}]$, have increases of $\sim 0.15$ to $\sim 0.25$ dex over the past $6-7 \mathrm{Gyr}$, respectively. These slopes agree with those of Spina et al. (2018), who find slopes of -0.029 and $-0.027 \mathrm{dex} \mathrm{Gyr}^{-1}$ for $\mathrm{Y}$ and $\mathrm{Zr}$, respectively. The elements of the second peak also show a slight increase with age, more pronounced for $[\mathrm{Ba} / \mathrm{Fe}]$ and $[\mathrm{Ce} / \mathrm{Fe}]$, and flatter for $[\mathrm{La} / \mathrm{Fe}]$ (see Table 6). Slopes for $\mathrm{Ba}$, at $-0.03 \mathrm{dex} \mathrm{Gyr}^{-1}$, agree with those found by da Silva et al. (2012) and Spina et al. (2018). In the case of La, which is measured only in giant stars, we have a much smaller sample of stars and therefore the measurement of the trend is less well constrained (see its relative error on the slope and its correlation coefficient in Table 6).

The growth in the $s$-process abundances in the youngest Galactic stellar populations has been reproduced by, for instance, the chemical evolution model of Maiorca et al. (2012), which was one of the first models that made an attempt to explain it. Their approach was to relate the $s$-process enhancement to a strong contribution to the production of these elements from low-mass stars, which start to contribute later in the lifetime of the Galaxy. In particular, they consider that the observed enhancements might be produced during the nucleosynthesis processes in the AGB phases of low-mass stars $\left(M<1.5 M_{\odot}\right)$ under the hypothesis that they release neutrons from the ${ }^{13} \mathrm{C}(\alpha, \mathrm{n}){ }^{16} \mathrm{O}$ reaction to create larger reservoirs of neutrons than in more massive AGB stars $\left(M>1.5 M_{\odot}\right)$. Later studies gave a physical explanation for the necessity of having larger reservoirs of neutrons, the so-called ${ }^{13} \mathrm{C}$ pocket, in low-mass stars. The extended ${ }^{13} \mathrm{C}$ pocket requires the existence of very efficient mixing episodes and the transport mechanisms most commonly
Table 6. Coefficients and Pearson indexes of the weighted linear fits for $[\mathrm{X} / \mathrm{Fe}]$ vs. stellar age (age $<8 \mathrm{Gyr})$.

\begin{tabular}{llll}
\hline \hline Element & Slope $\left(\mathrm{dex} \mathrm{Gyr}^{-1}\right)$ & Intercept $(\mathrm{dex})$ & $R$ \\
\hline Yttrium $[\mathrm{Y} / \mathrm{Fe}]$ & $-0.023 \pm 0.009$ & $0.13 \pm 0.03$ & -0.6 \\
Zirconium $[\mathrm{Zr} / \mathrm{Fe}]$ & $-0.038 \pm 0.013$ & $0.26 \pm 0.03$ & -0.5 \\
Barium $[\mathrm{Ba} / \mathrm{Fe}]$ & $-0.027 \pm 0.007$ & $0.09 \pm 0.02$ & -0.8 \\
Lanthanum $[\mathrm{La} / \mathrm{Fe}]$ & $-0.005 \pm 0.015$ & $0.10 \pm 0.04$ & -0.2 \\
Cerium $[\mathrm{Ce} / \mathrm{Fe}]$ & $-0.016 \pm 0.010$ & $0.10 \pm 0.03$ & -0.5 \\
\hline
\end{tabular}

adopted such as overshoot (Herwig 2000; Cristallo et al. 2009), gravity waves (Denissenkov et al. 2013; Battino et al. 2016), rotationally driven shear or thermohaline mixing do not suffice (Palmerini et al. 2011); but magnetic buoyancy can explain the process (Busso et al. 2007; Nordhaus et al. 2008; Trippella et al. 2016; Trippella \& La Cognata 2017).

However, in the framework of a common production of the second peak $s$-process elements, it is difficult to explain the difference in the steeper observed slope of $[\mathrm{Ba} / \mathrm{Fe}]$ as a function of stellar age with those those of $[\mathrm{La} / \mathrm{Fe}]$ and $[\mathrm{Ce} / \mathrm{Fe}]$, although their intercepts indicate similar maximum global enrichment $(\sim 0.1 \mathrm{dex})$ for all elements, in agreement with their similar nucleosynthesis. In addition, the maximum abundances of $[\mathrm{Ba} / \mathrm{Fe}]$ reached in the youngest open clusters is higher than $[\mathrm{La} / \mathrm{Fe}]$ and $[\mathrm{Ce} / \mathrm{Fe}]$. A larger enrichment of $\mathrm{Ba}$ compared to $\mathrm{La}$ is difficult to reconcile considering similar contributions to both elements by $s$ - and $r$-processes. Mishenina et al. (2015) discussed several possibilities to explain $[\mathrm{Ba} / \mathrm{La}]$ in young open clusters, which can reach $\sim 0.15$ dex. They proposed an additional contribution from an intermediate neutron-capture process, the so-called $i$-process. The $i$-process is characterised by neutron densities of the order of $10^{15}$ neutrons $\mathrm{cm}^{-3}$ and is triggered by the mixing or ingestion of $\mathrm{H}$ in He-burning 


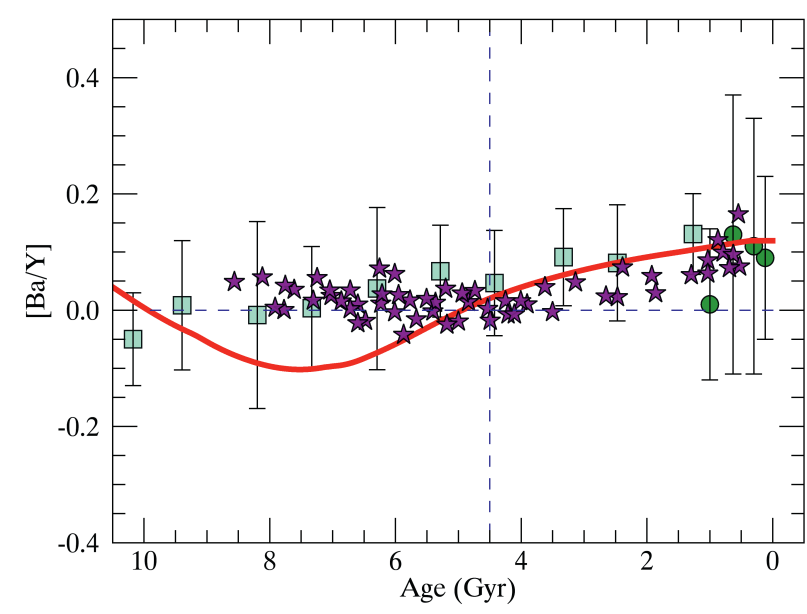

Fig. 10. Abundance ratios $[\mathrm{Ba} / \mathrm{Y}]$ vs. age in cluster (green circles) and thin-disc stars (cyan squares, bins of $1 \mathrm{Gyr}$ ) in the radial range $6.8 \mathrm{kpc}<R_{\mathrm{GC}}<8.2 \mathrm{kpc}$ and $-0.1<[\mathrm{Fe} / \mathrm{H}]<+0.1$, and in the solar twin stars of Spina et al. (2018) (purple stars). The model curve of Maiorca et al. (2012) is shown in red.

stellar layers (Cowan \& Rose 1977; Bertolli et al. 2013). The $i$-process can potentially explain this larger ratio and the higher $[\mathrm{Ba} / \mathrm{La}]$ in the youngest objects might suggest that the $i$-process contribution is becoming more relevant in the most recent few Gyr.

\subsection{The s-process relative indicator $[\mathrm{Ba} / \mathrm{Y}]$}

In Fig. 10 we show $[\mathrm{Ba} / \mathrm{Y}]$ versus stellar age. $\mathrm{Y}$ and $\mathrm{Ba}$ belong to the first and second peak, respectively, and therefore they allow us to estimate the relative weight of the production of light and heavy $s$-process elements with time. We have chosen these two elements because they are measured in many more stars than $\mathrm{Zr}, \mathrm{La}$, and $\mathrm{Ce}$. In the plot, we compare our results with the sample of solar twin stars of Spina et al. (2018). To do that, we have selected thin-disc stars and open clusters with the same characteristics as the sample chosen by Spina et al. (2018): $6.8 \mathrm{kpc}<R_{\mathrm{GC}}<8.2 \mathrm{kpc}$ and $-0.1<[\mathrm{Fe} / \mathrm{H}]<+0.1$. The two samples are in very good agreement; both indicate for $[\mathrm{Ba} / \mathrm{Y}]$ an increase from about $\sim 6 \mathrm{Gyr}$ to the youngest ages. The oldest stars show a slight growth of $[\mathrm{Ba} / \mathrm{Y}]$ together with an increasing dispersion. In this figure, we have also plotted the results of the model of Maiorca et al. (2012) for the time evolution of [Y/Ba]. The model results are in qualitative agreement, whilst it predicts a lower $[\mathrm{Ba} / \mathrm{Y}]$ for ages of more than $\sim 6 \mathrm{Gyr}$. As already noticed by Spina et al. (2018), the behaviour of [Ba/Y] versus stellar age is directly related to dependence of the AGB star yields on metallicity. The interplay during the chemical evolution of the Milky Way between very low-mass AGB stars, with enhanced reservoirs of ${ }^{13} \mathrm{C}$, and more massive AGB stars with the usual ${ }^{13} \mathrm{C}$ pocket, might produce the observed behaviour of $[\mathrm{Ba} / \mathrm{Y}]$ (see, e.g. Fig. 3 of Maiorca et al. 2012). The observational result can be explained also by the AGB models of Karakas \& Lugaro (2016), which predict that the [hs/ls] ratio in the winds of a typical AGB star is -0.026 at solar metallicity and +0.320 at halfsolar metallicity.

In Fig. 11, we expand the study of the behaviour of $[\mathrm{Ba} / \mathrm{Y}]$ versus stellar age to different radial and metallicity bins. We basically divide the sample into three radial ranges: the solar neighbourhood $6.2 \mathrm{kpc}<R_{\mathrm{GC}}<8.2 \mathrm{kpc}$, the inner disc $R_{\mathrm{GC}}<6.2 \mathrm{kpc}$, and the outer disc $R_{\mathrm{GC}}>8.2 \mathrm{kpc}$. Each radial range is then di- vided into metallicity bins: below solar $[\mathrm{Fe} / \mathrm{H}]<-0.1$, solar $-0.1<[\mathrm{Fe} / \mathrm{H}]<+0.1$, and super solar $[\mathrm{Fe} / \mathrm{H}]>+0.1$. The relative weight of the $s$-process indicator $[\mathrm{Ba} / \mathrm{Y}]$ clearly depends on the Galactocentric position, and therefore on the infall timescales, star formation history, and the metallicity. The growth of $[\mathrm{Ba} / \mathrm{Y}]$ with stellar ages is present in the solar neighbourhood, but it is less clear and less constrained in the inner disc and in the outer disc. The metallicity dependence is very strong, with $[\mathrm{Ba} / \mathrm{Y}]$ usually higher in the $[\mathrm{Fe} / \mathrm{H}]<-0.1$ range, and decreasing towards higher metallicities. All this information is new, and was not available at the time of previous studies. It provides important constraints for the study of $s$-process production.

\section{Summary and conclusions}

Taking advantage of the large and homogeneous sample of stellar parameters and elemental abundances of Gaia-ESO IDR5, we study the abundances of five $s$-process elements in the thin- and the thick-disc stars and in the population of open clusters. We compute statistical ages for field stars and perform membership analysis of stars in open clusters, computing the median abundances of each cluster. We estimate the effect of evolutionary stages in the $s$-process abundances by comparing giant and dwarf stars, which are members of the same clusters, and we do not find any remarkable difference. We identify the abundance ratio patterns versus $[\mathrm{Fe} / \mathrm{H}]$ of both field and open cluster stars, and their behaviour versus stellar ages. Using our large and homogenous sample of open clusters, thin- and thick-disc stars, spanning an age range larger than $10 \mathrm{Gyr}$, and selecting a sample of clusters and field stars in the solar ring, we confirm an increase of the five analysed $s$-process abundances towards young ages. The trend is more clear for $[\mathrm{Y} / \mathrm{Fe}],[\mathrm{Zr} / \mathrm{Fe}]$ and $[\mathrm{Ba} / \mathrm{Fe}]$, while it has a lower correlation coefficient for $[\mathrm{La} / \mathrm{Fe}]$ and $[\mathrm{Ce} / \mathrm{Fe}]$, perhaps because of the limited number of stars in which these elements are measured. Clusters located in the inner and outer disc have different abundance ratios with respect to those of the solar neighbourhood.

The global growth of the $s$-process abundance ratio with time can be explained by a strong contribution to the production of these elements from low-mass stars, which start to contribute later in the lifetime of the Galaxy (see, e.g. Maiorca et al. 2012). However, the differences in the maximum values reached in open clusters for $\mathrm{Ba}$ and $\mathrm{La}$ point to the necessity of an additional production mechanism for $\mathrm{Ba}$ and they might be explained by the occurrence of the $i$-process at later epochs in the evolution of the Galaxy (e.g. Mishenina et al. 2015). The new observations of Gaia-ESO have confirmed with a large statistical sample the behaviour of $s$-process elements in the solar neighbourhood, and now they give us the possibility to investigate the interplay among the different neutron-capture processes, the metallicity dependence of their stellar yields, and the physical process involved in the creation of this important group of elements in different parts of our Galaxy.

Acknowledgements. We are grateful to the referee for her/his comments and suggestions, which improved the quality of the paper. These data products have been processed by the Cambridge Astronomy Survey Unit (CASU) at the Institute of Astronomy, University of Cambridge, and by the FLAMES/UVES reduction team at INAF/Osservatorio Astrofisico di Arcetri. These data have been obtained from the Gaia-ESO Survey Data Archive, prepared and hosted by the Wide Field Astronomy Unit, Institute for Astronomy, University of Edinburgh, which is funded by the UK Science and Technology Facilities Council. This work was partly supported by the European Union FP7 programme through ERC grant number 320360 and by the Leverhulme Trust through grant RPG-2012-541. We acknowledge the support from INAF and Ministero dell' Istruzione, dell' Università' e della Ricerca (MIUR) in the form of the 

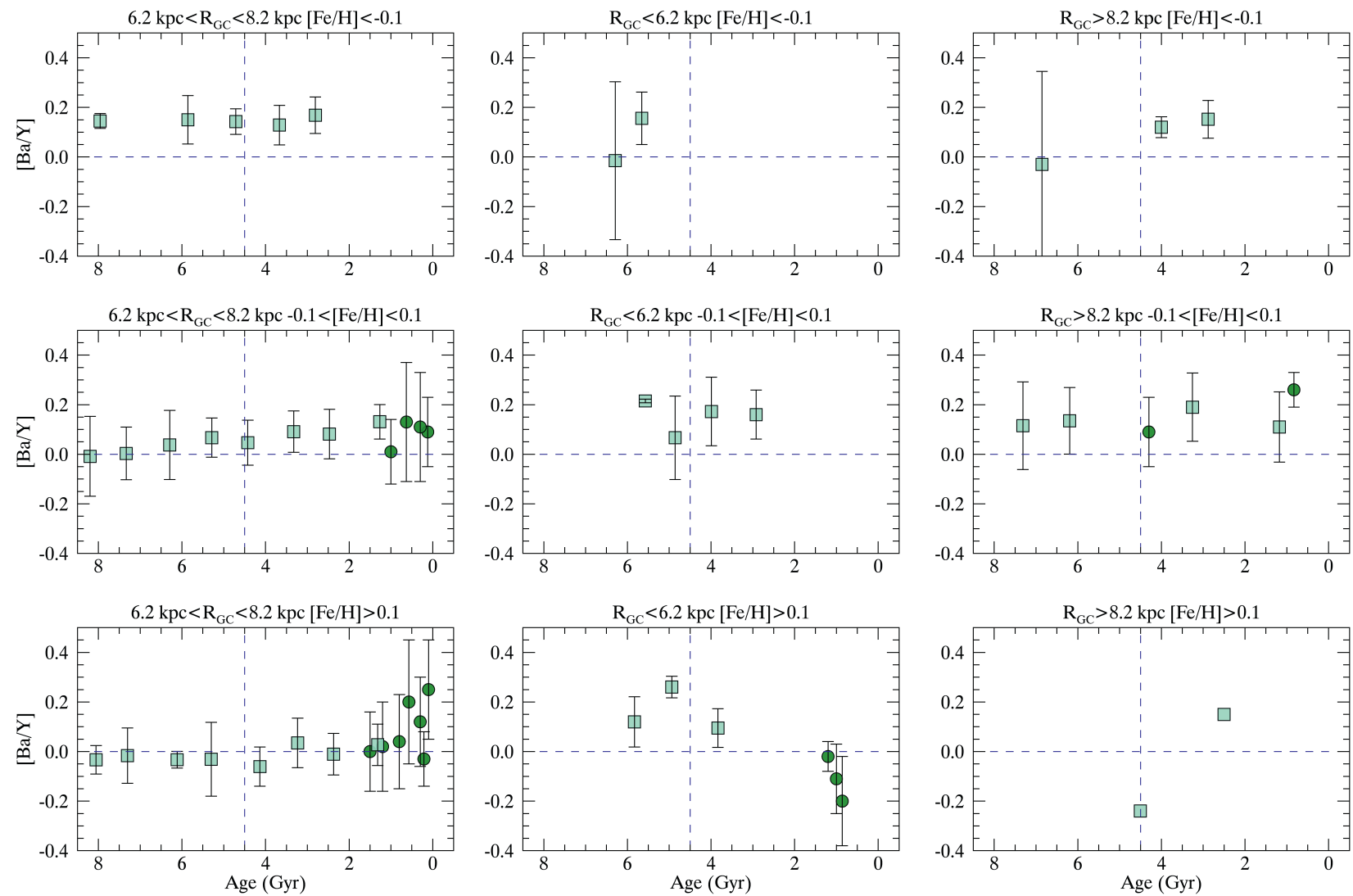

Fig. 11. Abundance ratios $[\mathrm{Ba} / \mathrm{Y}]$ vs. age in cluster and thin-disc stars (symbols as in Fig. 10) for the three radial ranges: $6.2 \mathrm{kpc}<R_{\mathrm{GC}}<8.2 \mathrm{kpc}$ (left panels), $R_{\mathrm{GC}}<6.2 \mathrm{kpc}$ (central panels) and $R_{\mathrm{GC}}>8.2 \mathrm{kpc}$ (right panels). For each radial bin, we divide the plot into three metallicity ranges: below solar $[\mathrm{Fe} / \mathrm{H}]<-0.1$ (panels in the upper row), solar $-0.1<[\mathrm{Fe} / \mathrm{H}]<+0.1$ (panels in the central row) and super solar $[\mathrm{Fe} / \mathrm{H}]>+0.1$ (panels in the lower row).

grant "Premiale VLT 2012". The results presented here benefit from discussions held during the Gaia-ESO workshops and conferences supported by the ESF (European Science Foundation) through the GREAT Research Network Programme. F.J.E. acknowledges financial support from ASTERICS project (ID:653477, H2020-EU.1.4.1.1. - Developing new world-class research infrastructures). S. D. acknowledges support from Comité Mixto ESO-GOBIERNO DE CHILE. AB thanks for support from the Millenium Science Initiative, Chilean Ministry of Economy. This project has received funding from the European Union's Horizon 2020 research and innovation programme under the Marie Sklodowska-Curie grant agreement No 664931. E.D.M., V.A. and S.G.S acknowledge the support from Fundação para a Ciência e a Tecnologia (FCT, Portugal) through the research grant through national funds and by FEDER through COMPETE2020 by grants UID/FIS/04434/2013 \& POCI-01-0145FEDER-007672, PTDC/FIS-AST/1526/2014 \& POCI-01-0145-FEDER-016886 and PTDC/FIS-AST/7073/2014 \& POCI-01-0145-FEDER-016880. E.D-M. V.A. and S.G.S also acknowledge support from FCT through Investigador FCT contracts nr. IF/00849/2015/CP1273/CT0003, IF/00650/2015/CP1273/CT0001 and IF/00028/2014/CP1215/CT0002. T.B. was supported by the project grant "The New Milky" from the Knut and Alice Wallenberg foundation. T.M acknowledges support provided by the Spanish Ministry of Economy and Competitiveness (MINECO) under grant AYA-2017-88254-P. R.S. acknowledges support from the Polish Ministry of Science and Higher Education. E.J.A. acknowledges financial support from MINECO, Spain, and FEDER funds through grant AYA2016-75931-C2-1-P.

\section{References}

Adibekyan, V. Z., Santos, N. C., Sousa, S. G., \& Israelian, G. 2011, A\&A, 535, L11

Alonso-Santiago, J., Negueruela, I., Marco, A., et al. 2017, MNRAS, 469, 1330 Asplund, M., Grevesse, N., Sauval, A. J., \& Scott, P. 2009, ARA\&A, 47, 481 Battino, U., Pignatari, M., Ritter, C., et al. 2016, ApJ, 827, 30

Battistini, C., \& Bensby, T. 2016, A\&A, 586, A49

Bensby, T., Feltzing, S., \& Oey, M. S. 2014, A\&A, 562, A71

Bertelli Motta, C., Pasquali, A., Richer, J., et al. 2018, MNRAS, 478, 425
Bertolli, M. G., Herwig, F., Pignatari, M., \& Kawano, T. 2013, ArXiv e-prints [arXiv:1310.4578]

Bisterzo, S., Travaglio, C., Gallino, R., Wiescher, M., \& Käppeler, F. 2014, ApJ, 787, 10

Bisterzo, S., Travaglio, C., Wiescher, M., Käppeler, F., \& Gallino, R. 2017, ApJ, 835, 97

Bragaglia, A., \& Tosi, M. 2006, AJ, 131, 1544

Bressan, A., Marigo, P., Girardi, L., et al. 2012, MNRAS, 427, 127

Burbidge, E. M., Burbidge, G. R., Fowler, W. A., \& Hoyle, F. 1957, Rev. Mod Phys., 29, 547

Busso, M., Gallino, R., \& Wasserburg, G. J. 1999, ARA\&A, 37, 239

Busso, M., Wasserburg, G. J., Nollett, K. M., \& Calandra, A. 2007, ApJ, 671, 802

Cantat-Gaudin, T., Vallenari, A., Zaggia, S., et al. 2014, A\&A, 569, A17

Carraro, G., Geisler, D., Moitinho, A., Baume, G., \& Vázquez, R. A. 2005, A\&A, 442,917

Carraro, G., Janes, K. A., Costa, E., \& Méndez, R. A. 2006, MNRAS, 368, 1078 Cignoni, M., Beccari, G., Bragaglia, A., \& Tosi, M. 2011, MNRAS, 416, 1077 Clem, J. L., Landolt, A. U., Hoard, D. W., \& Wachter, S. 2011, AJ, 141, 115

Côté, B., Fryer, C. L., Belczynski, K., et al. 2018, ApJ, 855, 99

Cowan, J. J., \& Rose, W. K., 1977, ApJ, 212, 149

Cristallo, S., Straniero, O., Gallino, R., et al. 2009, ApJ, 696, 797

da Silva, R., Porto de Mello, G. F., Milone, A. C., et al. 2012, A\&A, 542, A84

Delgado Mena, E., Tsantaki, M., Adibekyan, V. Z., et al. 2017, A\&A, 606, A94

Dell'Omodarme, M., Valle, G., Degl'Innocenti, S., \& Prada Moroni, P. G. 2012 , A\&A, 540, A26

Denissenkov, P. A., Herwig, F., Truran, J. W., \& Paxton, B. 2013, ApJ, 772, 37

Dias, W. S., Alessi, B. S., Moitinho, A., \& Lépine, J. R. D. 2002, A\&A, 389,871

Donati, P., Bragaglia, A., Cignoni, M., Cocozza, G., \& Tosi, M. 2012, MNRAS, 424, 1132

Donati, P., Cantat Gaudin, T., Bragaglia, A., et al. 2014, A\&A, 561, A94

D’Orazi, V., Magrini, L., Randich, S., et al. 2009, ApJ, 693, L31

Feltzing, S., Howes, L. M., McMillan, P. J., \& Stonkutè, E. 2017, MNRAS, 465, L109

Friel, E. D., Donati, P., Bragaglia, A., et al. 2014, A\&A, 563, A117 
Gaia Collaboration (Prusti, T., et al.) 2016, A\&A, 595, A1

Gallino, R., Arlandini, C., Busso, M., et al. 1998, ApJ, 497, 388

Gilmore, G., Randich, S., Asplund, M., et al. 2012, The Messenger, 147, 25

González Hernández, J. I., Israelian, G., Santos, N. C., et al. 2010, ApJ, 720, 1592

Grevesse, N., Asplund, M., \& Sauval, A. J. 2007, Space Sci. Rev., 130, 105

Herwig, F. 2000, A\&A, 360, 952

Jacobson, H. R., \& Friel, E. D. 2013, AJ, 145, 107

Jacobson, H. R., Friel, E. D., \& Pilachowski, C. A. 2011, AJ, 141, 58

Jacobson, H. R., Friel, E. D., Jilkova, L., et al. 2016, A\&A, 591, A37

Karakas, A. I., \& Lugaro, M. 2016, ApJ, 825, 26

Kordopatis, G., Recio-Blanco, A., de Laverny, P., et al. 2011, A\&A, 535, A 107

Kordopatis, G., Wyse, R. F. G., Gilmore, G., et al. 2015, A\&A, 582, A122

Liu, F., Asplund, M., Yong, D., et al. 2016, MNRAS, 463, 696

Maiorca, E., Randich, S., Busso, M., Magrini, L., \& Palmerini, S. 2011, ApJ, 736, 120

Maiorca, E., Magrini, L., Busso, M., et al. 2012, ApJ, 747, 53

Magrini, L., Sestito, P., Randich, S., \& Galli, D. 2009, A\&A, 494, 95

Magrini, L., Randich, S., Donati, P., et al. 2015, A\&A, 580, A85

Magrini, L., Randich, S., Kordopatis, G., et al. 2017, A\&A, 603, A2

Mashonkina, L., \& Gehren, T. 2001, A\&A, 376, 232

Matteucci, F., Romano, D., Arcones, A., Korobkin, O., \& Rosswog, S. 2014, MNRAS, 438, 2177

Mermilliod, J.-C., Clariá, J. J., Andersen, J., Piatti, A. E., \& Mayor, M. 2001, A\&A, 375, 30

Mishenina, T., Korotin, S., Carraro, G., Kovtyukh, V. V., \& Yegorova, I. A. 2013 MNRAS, 433, 1436

Mishenina, T., Pignatari, M., Carraro, G., et al. 2015, MNRAS, 446, 3651

Modigliani, A., Mulas, G., Porceddu, I., et al. 2004, The Messenger, 118, 8

Nissen, P. E. 2016, A\&A, 593, A65

Nissen, P. E., Silva Aguirre, V., Christensen-Dalsgaard, J., et al. 2017, A\&A, 608, A112

Nordhaus, J., Busso, M., Wasserburg, G. J., Blackman, E. G., \& Palmerini, S. 2008, ApJ, 684, L29

Önehag, A., Gustafsson, B., \& Korn, A. 2014, A\&A, 562, A102

Overbeek, J. C., Friel, E. D., \& Jacobson, H. R. 2016, ApJ, 824, 75

Pagel, B. E. J., \& Tautvaisiene, G. 1997, MNRAS, 288, 108

Palmerini, S., Cristallo, S., Busso, M., et al. 2011, ApJ, 741, 26

Pancino, E., Lardo, C., Altavilla, G., et al. 2017, A\&A, 598, A5

Pasquini, L., Avila, G., Blecha, A., et al. 2002, The Messenger, 110, 1

Pasquini, L., Biazzo, K., Bonifacio, P., Randich, S., \& Bedin, L. R. 2008, A\&A 489, 677

Piatti, A. E., Clariá, J. J., Bica, E., Geisler, D., \& Minniti, D. 1998, AJ, 116,801

Pignatari, M., Gallino, R., Heil, M., et al. 2010, ApJ, 710, 1557

Raiteri, C. M., Gallino, R., Busso, M., Neuberger, D., \& Kaeppeler, F. 1993, ApJ, 419, 207

Raiteri, C. M., Villata, M., Gallino, R., Busso, M., \& Cravanzola, A. 1999, ApJ, 518, L91

Randich, S., Sestito, P., Primas, F., Pallavicini, R., \& Pasquini, L. 2006, A\&A, 450,557

Randich, S., Gilmore, G., \& Gaia-ESO Consortium 2013, The Messenger, 154,

Randich, S., Tognelli, E., Jackson, R., et al. 2018, A\&A 612, A99

Recio-Blanco, A., de Laverny, P., Kordopatis, G., et al. 2014, A\&A, 567, A5

Reddy, A. B. S., \& Lambert, D. L. 2017, ApJ, 845, 151

Sacco, G. G., Morbidelli, L., Franciosini, E., et al. 2014, A\&A, 565, A113

Salaris, M., Weiss, A., \& Percival, S. M. 2004, A\&A, 414, 163

Schlegel, D. J., Finkbeiner, D. P., \& Davis, M. 1998, ApJ, 500, 525

Seeger, P. A., Fowler, W. A., \& Clayton, D. D. 1965, ApJS, 11, 121

Sharma, S., Pandey, A. K., Ogura, K., et al. 2006, AJ, 132, 1669

Silva Aguirre, V., Lund, M. N., Antia, H. M., et al. 2017, ApJ, 835, 173

Smiljanic, R., Korn, A. J., Bergemann, M., et al. 2014, A\&A, 570, A122

Sneden, C., Cowan, J. J., \& Gallino, R. 2008, ARA\&A, 46, 241

Spina, L., Palla, F., Randich, S., et al. 2015, A\&A, 582, L6

Spina, L., Meléndez, J., Karakas, A. I., et al. 2016, A\&A, 593, A125

Spina, L., Meléndez, J., Karakas, A. I., et al. 2018, MNRAS, 474, 2580

Stonkutè, E., Koposov, S. E., Howes, L. M., et al. 2016, MNRAS, 460, 1131

Thielemann, F.-K., Arcones, A., Käppeli, R., et al. 2011, Prog. Part. Nucl. Phys., 66,346

Tognelli, E., Prada Moroni, P. G., \& Degl'Innocenti, S. 2011, A\&A, 533, A109

Travaglio, C., Galli, D., Gallino, R., et al. 1999, ApJ, 521, 691
Travaglio, C., Gallino, R., Arnone, E., et al. 2004, ApJ, 601, 864

Trippella, O., Busso, M., Palmerini, S., Maiorca, E., \& Nucci, M. C. 2016, ApJ, 818,125

Trippella, O., \& La Cognata, M. 2017, ApJ, 837, 41

Yong, D., Carney, B. W., \& Friel, E. D. 2012, AJ, 144, 95

Zhao, G., Mashonkina, L., Yan, H. L., et al. 2016, ApJ, 833, 225

1 INAF - Osservatorio Astrofisico di Arcetri, Largo E. Fermi, 5, 50125 Firenze, Italy

e-mail: laura@arcetri.astro.it

2 Monash Centre for Astrophysics, School of Physics and Astronomy, Monash University, VIC 3800, Australia

3 Department of Astronomy, Indiana University, Bloomington, IN, USA

4 Université Côte d'Azur, Observatoire de la Côte d'Azur, CNRS, Laboratoire Lagrange, France

5 Institute of Astronomy, Madingley Road, University of Cambridge, CB3 OHA, UK

6 Space Science Data Center - Agenzia Spaziale Italiana, Via del Politecnico SNC, 00133 Roma, Italy

7 INAF - Osservatorio di Astrofisica e Scienza dello Spazio, via Gobetti 93/3, 40129 Bologna, Italy

8 Astronomical Observatory, Institute of Theoretical Physics and Astronomy, Vilnius University, av. Saulètekio al. 3, 10257 Vilnius, Lithuania

9 Instituto de Astrofisica e ciencias do espaço - CAUP Universidade do Porto, Rua das Estrelas, 4150-762 Porto, Portugal

10 Departamento de Astrofísica, Centro de Astrobiología (INTACSIC), ESAC Campus, Camino Bajo del Castillo s/n, 28692 Villanueva de la Cañada, Madrid, Spain

11 INAF - Osservatorio Astronomico di Palermo, Piazza del Parlamento 1, 90134 Palermo, Italy

12 European Southern Observatory, Alonso de Cordova 3107 Vitacura, Santiago de Chile, Chile

13 Departamento de Ciencias Fisicas, Universidad Andres Bello, Fernandez Concha 700, Las Condes, Santiago, Chile

14 Lund Observatory, Department of Astronomy and Theoretical Physics, Box 43, 22100 Lund, Sweden

15 Astrophysics Group, Keele University, Keele, Staffordshire ST5 5BG, UK

16 INAF - Padova Observatory, Vicolo dell'Osservatorio 5, 35122 Padova, Italy

17 Instituto de Astrofísica de Andalucía-CSIC, Apdo. 3004, 18080 Granada, Spain

18 GEPI, Observatoire de Paris, CNRS, Université Paris Diderot, 5 Place Jules Janssen, 92190 Meudon, France

19 Department of Physics and Astronomy, Uppsala University, Box 516, 75120 Uppsala, Sweden

20 Laboratoire Lagrange (UMR7293), Université de Nice Sophia Antipolis, CNRS,Observatoire de la Côte d'Azur, CS 34229, 06304 Nice Cedex 4, France

21 Nicolaus Copernicus Astronomical Center, Polish Academy of Sciences, ul. Bartycka 18, 00-716 Warsaw, Poland

22 Instituto de Física y Astronomiía, Universidad de Valparaiíso, Chile

23 Millennium Nucleus "Núcleo Planet Formation", Universidad de Valparaíso, Chile

24 Dipartimento di Fisica e Astronomia, Università di Padova, Vicolo dell'Osservatorio 3, 35122 Padova, Italy

25 Departamento de Didáctica, Universidad de Cádiz, 11519 Puerto Real, Cádiz, Spain

26 INAF - Osservatorio Astrofisico di Catania, via S. Sofia 78, 95123 Catania, Italy

27 Núcleo de Astronomía, Facultad de Ingeniería, Universidad Diego Portales, Av. Ejército 441, Santiago, Chile

28 Instituto de Astrofísica de Canarias, 38205 La Laguna, Tenerife, Spain

29 Universidad de La Laguna, Department of Astrofísica, 38206 La Laguna, Tenerife, Spain 